\title{
CYCLES VITAUX ET PRODUCTION DES MICRASEMA PYRẼNẼENNES DANS LES MOUSSES D'EAU COURANTE
}

\author{
[Trichoptera, Brachycentridae]
}

\author{
par H. DÉCAMPs ${ }^{1}$ el M. LAFONT ${ }^{2}$.
}

Plusieurs populations des cinq espèces pyrénéennes de Micrasema sont étudiées dans des mousses d'eatı courante.

Micrasema morosum présente la plus large amplitude altitudinale. Son cycle vital dure un an, deux ans ou trois ans selon l'altitude. Les quatre autres Micrasema ont un cycle d'un an, avec de simples décalages des périodes de vol selon l'altitude.

Au cours du développement larvaire, on ne constate pas d'arrêt saisonnier dans l'alimentation. Les larves de premier stade se nourrissent d'algues périphytiques; aux autres stades, des fragments de mousses et des algues périphytiques sont indistinctement consommés. En présence d'une abondante couverture de mousses, les larves se développent presque exclusivement dans ce milieu. Dans le cas contraire, des déplacements peuvent être observés entre les pierres et les mousses.

Pour une même cohorte, le taux de croissance en poids sec moyen est relativement constant en basse altitude mais varie au cours du cycle vital en moyenne et en haute altitude. Ces variations se reproduisent d'une cohorte à la suivante. De même, les variations du taux de diminution des nombres se reproduisent, d'une manière semblable, d'une cohorte à la suivante.

Les trois méthodes utilisées pour calculer la production de deux espèces ont donné des résultats voisins. Des différences nettes apparaissent entre deux espèces de Micrasema en une même station de moyenne altitude. Pour une espèce donnée, la production varie également d'une cohorte à la suivante : l'origine de ces variations est à rechercher au niveau de la séquence nymphe adulte-ponte. En revanche, le rapport Production/Biomasse maximale paraît constant et voisin de ceux mentionnés pour d'autres espèces des Pyrénées centrales.

\section{Life cycles and production of Micrasema spp. (Trichoptera : Brachycentridae) in the mosses of Pyrenean streams.}

A study was made of several populations of five species of Micrasema living in the mosses of Pyrenean streams.

The life cycle of Micrasema morosum showed the greatest changes with altitude and took one, two or three years depending on altitude. The four

1. Laboratoire d'Hydrobiologie, Université Paul-Sabatier, 118, route de Narbonne, 31077, Toulouse Cedex.

2. C.T.G.R.E.F.. Institut de Sciences Naturelles, place Maréchal-Leclerc, 25000 Besançon. 
other species of Micrasema had a life cycle of one year with a simple shift in the flight period according to altitude.

There was no seasonal cessation of feeding in the larval stage. Larvae in the first instar fed on periphytic algae, but those in the other instars consi:med fragments of moss. When there was an abundant cover of mosses, the larvae were virtually confined to this habitat. In the opposite situation, there was some movement of larvae between the stones and the mosses.

For the same cohort, the rate of growth in mean dry weight was relatively constant at low altitude, but varied during the life cycle at average and high altitudes. These changes were repeated from one cohort to the next. The variations in the rates of decrease in numbers were also repeated from one cohort to the next.

The three methods used to calculate the production of two species gave similar results. There were clear differences between the production of the two species at the same station at average altitude. The production varied considerably between two successive cohorts of the same species : the reasons for these differences are probably to be found in the factors affecting the life cycle during the sequence : pupae-adults-eggs. Although production varied considerably between cohorts, the ratio of Production/Maximum Biomass was fairly constant and was similar to values obtained for other species in the central Pyrenees.

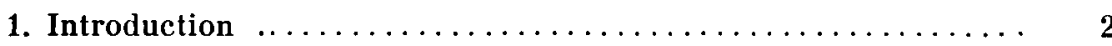

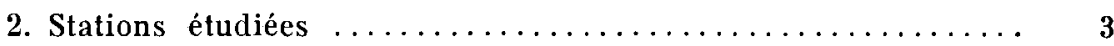

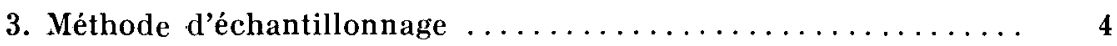

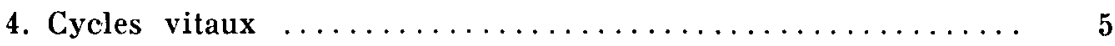

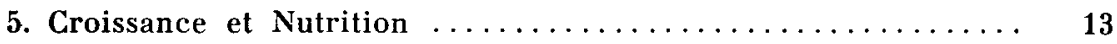

6. Fécondité et évolution des nombres $\ldots \ldots \ldots \ldots \ldots \ldots \ldots \ldots \ldots$

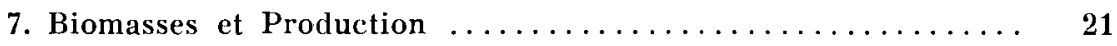

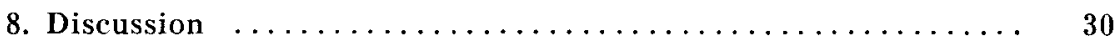

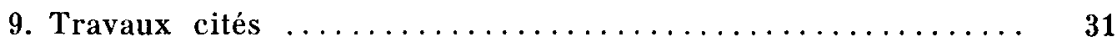

\section{1. - INTRODUCTION}

Cinq espèces de Micrasema vivent dans les Pyrénées : M. morosum (Mc. L.), M. longulum Mc. L., M. moestum (Hagen), M. difficile Mosely et $M$. minimum Mc. L. Les larves se développent dans des eaux courantes claires et bien oxygénées. Elles se remplacent régulièrement le long des réseaux hydrographiques mais peuvent cohabiter en certaines stations de moyenne altitude (Décamps 1968). Ces larves sont surtout abondantes dans les mousses.

Une clé de détermination permet la distinction directe des larves de Micrasema de la faune de France (Décamps 1970, Verneaux 1972). En ce qui concerne les mousses, des biomasses et productions ont été évaluées pour quelques espèces d'eau courante dans les Pyrénées (Dawson 1973).

Le présent travail porte sur plusieurs populations des cinq espèces de Micrasema vivant dans les mousses des ruisseaux pyré- 
néens. Les cycles vitaux sont comparés à différentes altitudes, de même que les croissances et les mortalités. Des biomasses et des productions sont estimées pour deux espèces récoltées en une même station.

\section{2. - LES STATIONS ETUDIEES}

Quatre stations ont été étudiées dans la vallée d'Aure : à $1940 \mathrm{~m}$ d'altitude (ruisseau affluent d'Estaragne), à $1590 \mathrm{~m}$ d'altitude (ruisseau d'Artigusse), à $1360 \mathrm{~m}$ d'altitude (ruisseau d'Espiaube) et à $550 \mathrm{~m}$ d'altitude (rivière Le Nistos). Une station a été étudiée dans la rivière Le Volp à $430 \mathrm{~m}$ d'altitude. Ces stations ont déjà été décrites en détail (Berthélemy 1966, Décamps $1967)$.

Nous précisons ici les espèces rencontrées (Micrasema et mousses), les températures observées et l'importance de la couverture de mousses.

(1) A $1940 \mathrm{~m}$ d'altitude, le ruisseau affluent d'Estaragne abrite Micrasema morosum. En hiver, sous la neige, la température de l'eau s'abaisse à $1{ }^{\circ} \mathrm{C}$; en été, elle demeure voisine de $4^{\circ} \mathrm{C}$ et peut atteindre $7{ }^{\circ} \mathrm{C}$ au mois d'août. Environ $7 \%$ du fond du ruisseau est recouvert de mousses (Dawson 1973) :

- Brachythecium rivulare B. S. G.;

- Cratoneurum commutatum (Hedw.) Roth.;

- Hygrohypnum molle (Dicks) Loeske.

(2) A $1590 \mathrm{~m}$ d'altitude, le ruisseau d'Artigusse abrite Micrasema morosum et $M$. difficile. Par suite de la proximité des sources, les températures varient entre 4 et $8^{\circ} \mathrm{C}$ au cours de l'année. Plus de $60 \%$ du fond est, en permanence, recouvert de mousses (Dawson 1973) :

- Fontinalis squamosa L. (ex Hedw.);

- Fissidens polyphyllus Wils.;

- Platyhypnidium riparioides (Hedw.) Dix.

(3) A $1360 \mathrm{~m}$ d'altitude, le ruisseau d'Espiaube abrite Micrasema morosum el, beaucoup plus rares, quelques larves de $\boldsymbol{M}$. difficile et $M$. minimum. Les températures varient entre 1 et $10^{\circ} \mathrm{C}$ en janvier, entre 8 et $14^{\circ} \mathrm{C}$ en août. Moins de $5 \%$ du fond est recouvert de mousses :

- Fissidens (Pachyfissidens) grandifrons (Brid. Limp.):

- Platyhypnidium riparioides (Hedw.) Dix. 
(4) A $550 \mathrm{~m}$ d'altitude, la rivière Le Nistos abrite les cinq espèces de Micrasema pyrénéennes: $M$. morosum, $M$. longulum, $M$. moestum, $M$. difficile et $M$. minimum. En 1969, les températures se sont abaissées à $5^{\circ} \mathrm{C}$ en hiver et n'ont pas dépassé $15^{\circ} \mathrm{C}$ en été. Entre 10 et $20 \%$ du fond est recouvert de mousses :

-- Brachythecium rivulare B. S. G.;

- Fissidens (Pachyfissidens) grandifrons (Brid.) Limp.;

- Platyhypnidium riparioides (Hedw.) Dix.;

- Chiloscyphus polyanthus L. (Corda) var. rivularis (Schrade) (Hépatique).

(5) A $430 \mathrm{~m}$ d'altitude, la rivière Le Volp abrite Micrasema moestum. En 1969 et 1970, les températures se sont abaissées à $6{ }^{\circ} \mathrm{C}$ en hiver et élevées à $20^{\circ} \mathrm{C}$ en été. Entre 5 et $10 \% \mathrm{du}$ fond est recouvert de mousses :

-- Brachythecium rivulare B.S. G.;

- Cinclidotus fontinaloides (Hedw.) P. Beauv.;

- Fontinalis antipyrethica L.;

- Platyhypnidium riparioides (Hedw.) Dix.;

- Cratoneuron filicinum (Hedw.) Roth.

\section{3. - MÉTHODE D'ÉCHANTILLONNAGE}

L'échantillonnage des mousses en eau courante peut être réalisé en prenant comme unité de relevé un volume de mousses (Frost 1942, Hynes 1961) ou une aire donnée (Minckley 1963, Kamler et Riedel 1960).

Dans ce travail, chaque échantillon comprend un volume égal à $900 \mathrm{~cm}^{3}$ de mousses. Ces mousses ont été prélevées par petits paquets pris au hasard dans l'aire à échantillonner. Les Micrasema, solidement attachées aux mousses leur servant de support, ont toute chance d'être ainsi recueillies. Cette méthode est commode sur le terrain mais ne permet pas de préciser les limites de confiance des nombres obtenus. Les paquets de mousses pris au hasard, et constituant les relevés (ou unités d'échantillonnage) sont en effet trop petits pour que leur volume soit déterminé avec précision. L'énorme travail de tri nécessaire dans le cas des mousses ne permet pas d'augmenter le volume de ces relevés. Cependant, chaque échantillon comprenant plusieurs petits paquets de mousses (entre 10 et 20 ), le nombre de Micrasema ainsi recueillies dans $900 \mathrm{~cm}^{3}$ de mousses peut être considéré comme indiquant une estimation proche de la moyenne valable pour l'ensemble de la population. 
Notre élude comparée des cycles vitaux, des croissances et des mortalités repose sur cette base $(\S 4$ à 6$)$. Les biomasses et productions ont été évaluées en reliant ces volumes aux surfaces correspondantes du fond ( $\S 7$ ). Pour le ruisseau d'Artigusse notamment (§ 7.1.), le poids sec moyen de $900 \mathrm{~cm}^{3}$ de mousses a été déterminé après passage à l'étuve à $105^{\circ} \mathrm{C}$ pendant 12 heures, puis comparé au poids sec moyen $/ \mathrm{m}^{2}$ tel qu'il est indiqué par Dawson (1973) pour cette station.

\section{4. - CYCLES VITAUX}

\section{1. - Présentation des résultats.}

Les stades larvaires ont été distingués par mesure de la largeur des capsules céphaliques. Cette méthode s'est révélée sûre dans le cas des Micrasema: les séparations entre stades sont nettes, les ambiguïtés rares (fig. $1 \mathrm{~A}$ ).

Dans les figures 3 à 6 , chacun des 5 stades larvaires est localisè sur l'axe des ordonnées en fonction de son poids sec moyen. Pour les 5 espìces étudiées, ces poids secs moyens sont regroupés (fig. 2 ). Les 4 premiers stades larvaires ont été pesés par groupes de 20 à 50 individus. Les stades $\mathrm{V}$ ont été pesés individuellement et, pour chaque espèce, la moyenne est accompagnée des valeurs extrêmes observées (fig. 2) sur des lots d'au moins 30 individus choisis au hasard. Ces pesées ont été effectuées sur des larves conservées en alcool à $70^{\circ}$, sorties de leur fourreau puis séchées à l'étuve à $60^{\circ} \mathrm{C}$ pendant 12 heures (Edmondson et Winberg 1971).

Les segments verticaux en traits fins (fig. 3 à 6) unissent les stades présents à la date considérée. Les poids moyens de chaque cohorte sont reliés par un trait plus épais. Ils sont obtenus à partir des poids moyens de chaque stade (fig. 2). Par exemple, pour Micrasema morosum à la rivière le Nistos $(550 \mathrm{~m})$, on observe en janvier 1969 ( $f$ ig. 4$): 6$ stades III $(0,1 \mathrm{mg}), 18$ stades IV $(0,2 \mathrm{mg})$ et 24 stades $\mathrm{V}(0,8 \mathrm{mg})$, soit 48 individus d'un poids total égal à $(6 \times 0,1)+(18 \times 0,2)+(24 \times 0,8)=23,4 \mathrm{mg}$, et d'un poids moyen égal à $23,4 / 48=0,49 \mathrm{mg}$.

La distinction des cohortes, parfois délicate lorsque deux ou trois cohortes coexistent dans un prélèvement (fig. $1 \mathrm{~B}$ ), peut être facilitée par la présence de hiatus. En certains cas, des individus de stade $\mathrm{V}$ appartenant à des cohortes distinctes coexistent dans un même prélèvement. La séparation est alors possible grâce aux différences d'aspect des larves : les plus petites (jeune cohorte) se distinguent nettement des plus grandes (ancienne cohorte). Dans les figures 3 à 6 , chaque cohorte est désignée par l'année de dépôt des œufs, écrite entre parenthèses. 

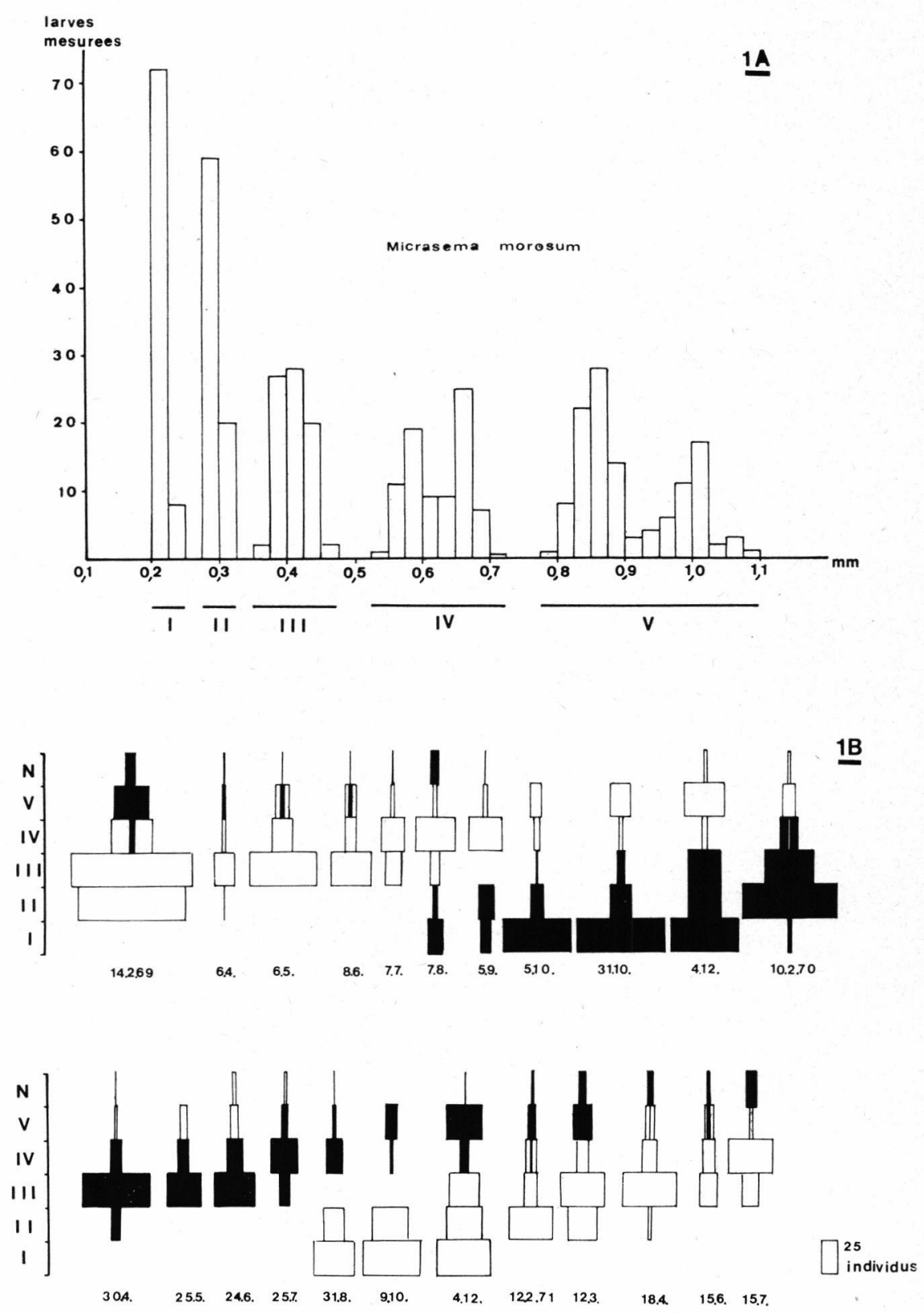

Fig. 1. - Exemples de distinction A) des stades larvaires par mesure de de la largeur de la capsule céphalique (en haut) et B) des cohortes dans le cas d'une population dont le cycle est supérieur à un an (en bas): Micrasema morosum à $1500 \mathrm{~m}$ (ruisseau d'Artigusse). 


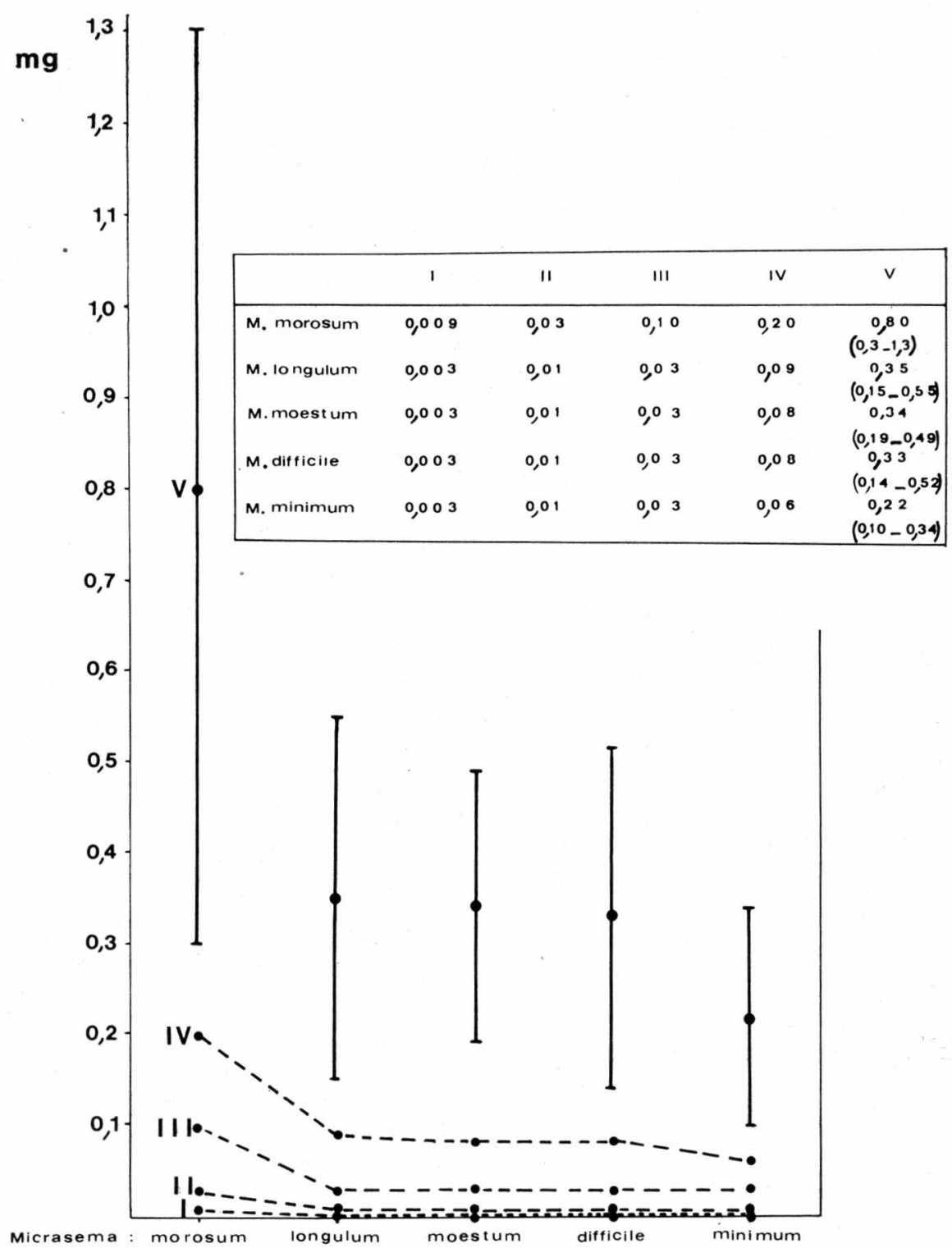

Fig. 2. - Poids moyens des différents stades des Micrasema pyrénéennes et valeurs limites observées pour les stades V. 

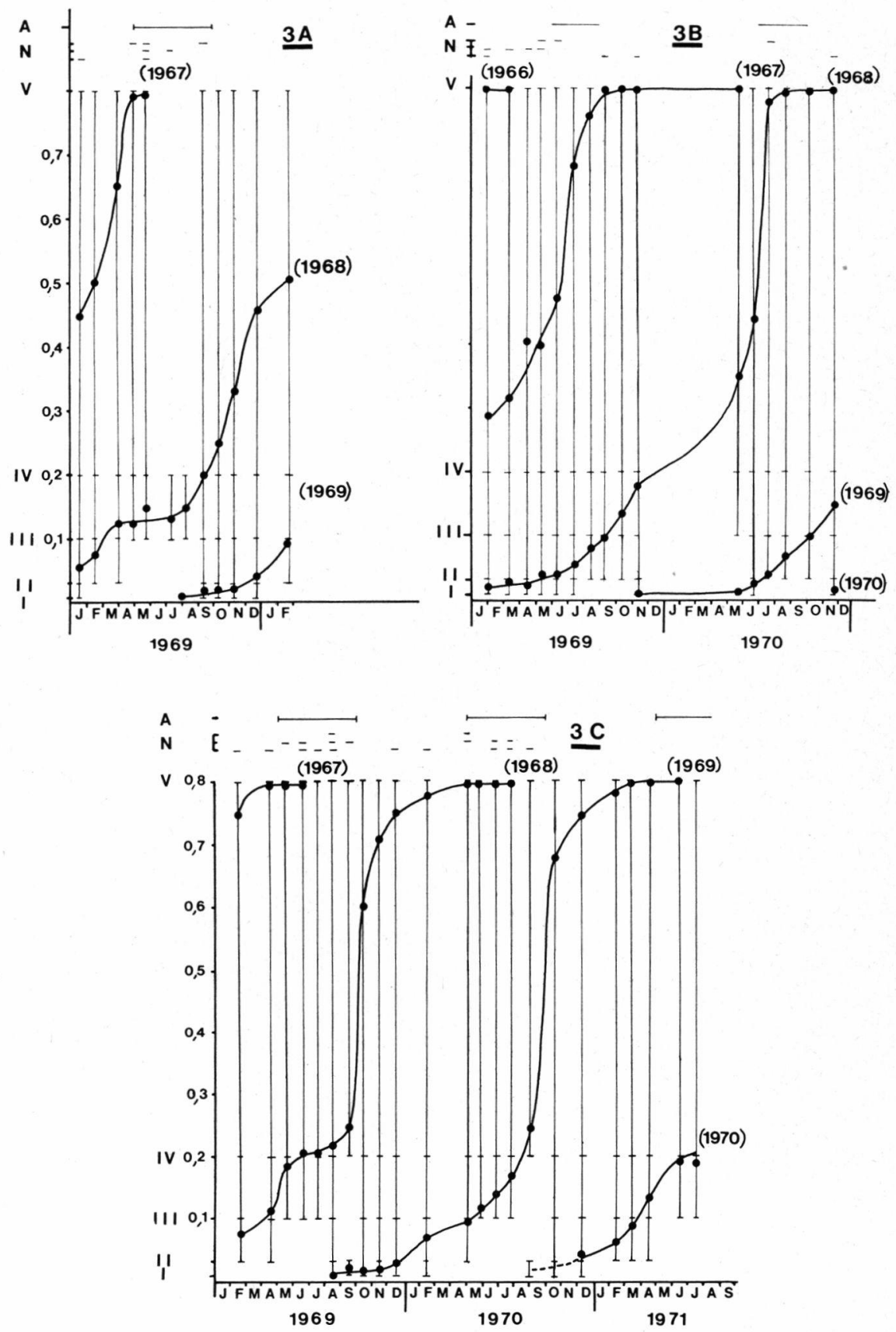

FIG. 3. - Cycles vitaux de Micrasema morosum a) à $1360 \mathrm{~m}$ (ruisseau d'Espiaube); b) à $1940 \mathrm{~m}$ (affluent du ruisseau d'Estaragne); c) à $1590 \mathrm{~m}$ (ruisseau d'Artigusse). A : Adultes, N : Nymphes. 

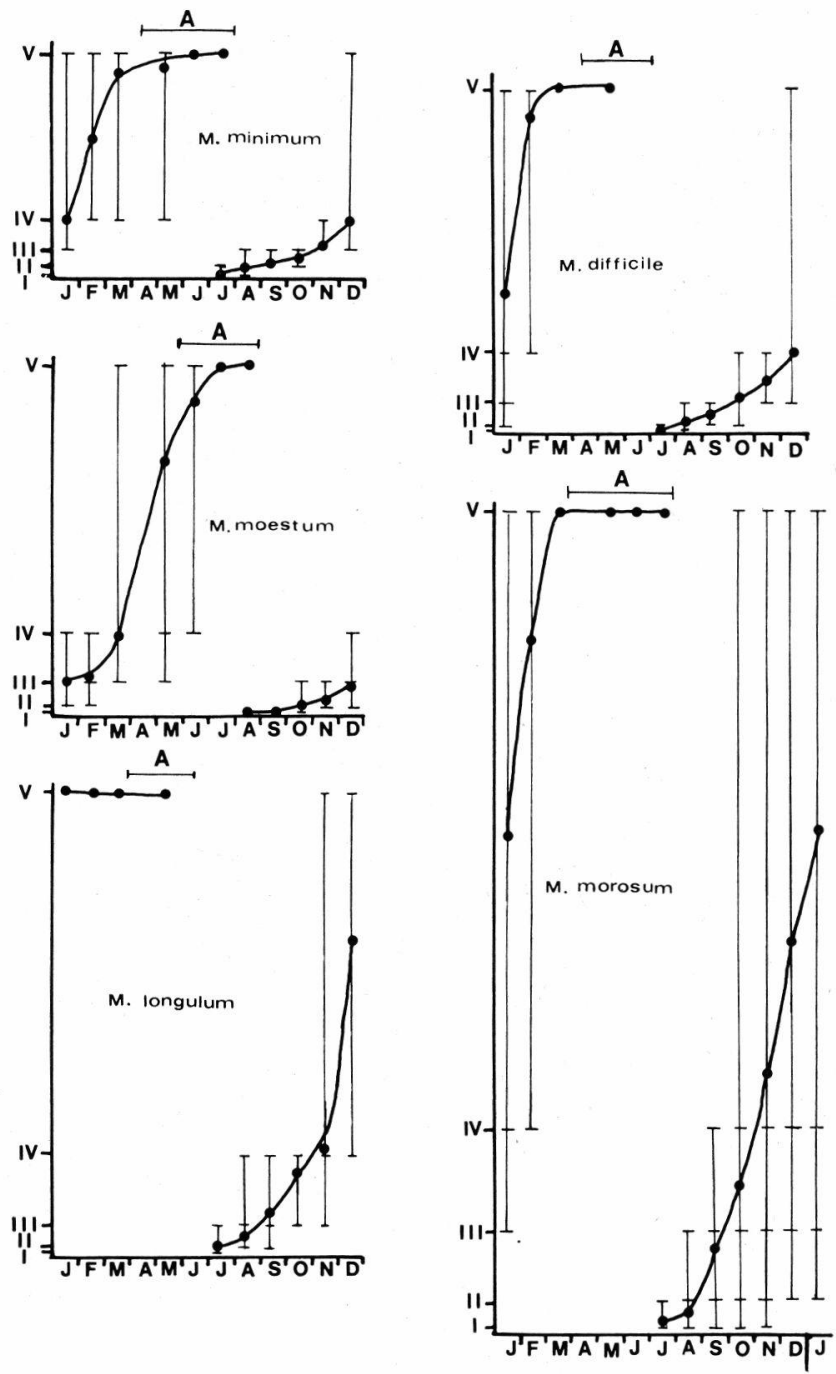

Fıg. 4. - Cycles vitaux des cinq espèces de Micrasema présentes dans la rivière le Nistos à $550 \mathrm{~m}$ d'altitude (année 1969). 


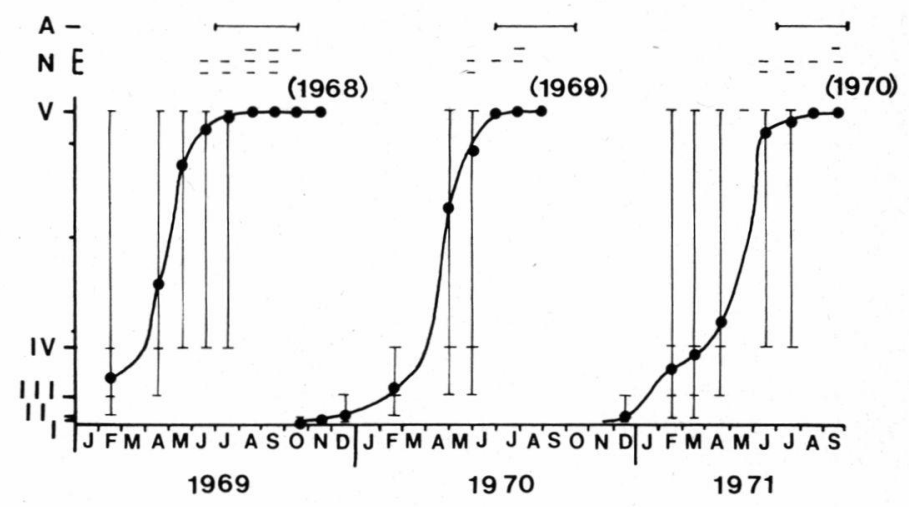

Fig. 5. - Cycle vital de Micrasema difficile à $1590 \mathrm{~m}$ (ruisseau d'Artigusse).

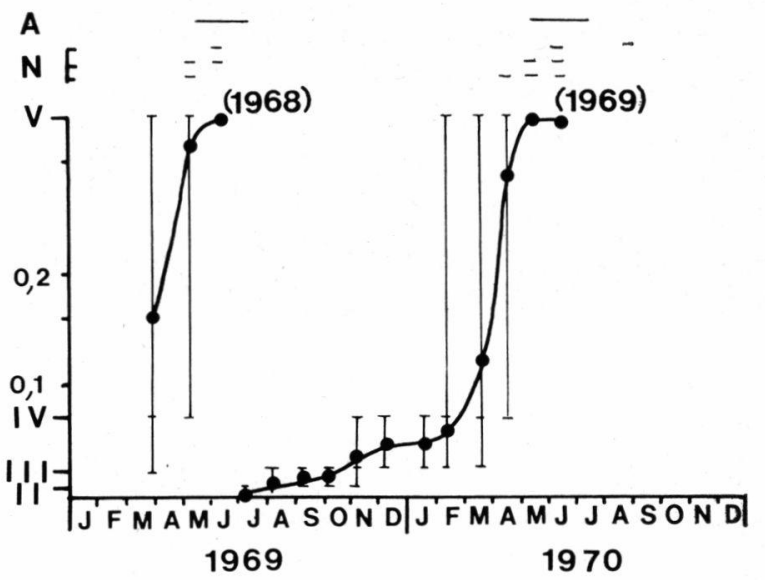

Fig. 6. - Cycle vital de Micrasema moestum à $430 \mathrm{~m}$ (rivière Le Volp). 
Trois états de maturité ont été distingués pour les nymphes. Les prénymphes $N_{1}$ correspondent à des larves de stade $V$ ayant fermé les extrémités de leur fourreau. Les nymphes $\mathrm{N}_{2}$ ont repoussé leur dépouille larvaire au fond de leur fourreau; ces nymphes présentent une couleur uniformément claire; les génitalia, plus ou moins individualisés, ne sont pas sclérifiés. Les nymphes $\mathbf{N}_{3}$ ont une coloration semblable à celle de l'adulte: les fourreaux alaires, la tête et lc thorax bruns contrastent avec l'abdomen verdâtre; les génitalia sont sclérifiés.

\section{2. - Cycle vital de Micrasema morosum à différentes altitudes.}

A $550 \mathrm{~m}$ d'altitude (rivière le Nistos), le cycle vital dure une année (fig. 4). Les éclosions s'échelonnent de juillet à novembre. La plus grande partie de la croissance a lieu en automne et en hiver. Tous les stades larvaires sont présents dans les prélèvements d'octobre et de novembre. En mars, toutes les larves ont atteint ou dépassé le stade $V$. Des nymphes $N_{1}$ apparaissent dès janvier. Les premiers adultes s'envolent en avril.

A $1360 \mathrm{~m}$ d'altitude (ruisseau d'Espiaube), le cycle vital dure deux années (fig. $3 \mathrm{~A}$ ). Les éclosions s'échelonnent d'août à janvier. La croissance, ralentie en été, s'étend sur les autres saisons de l'année. La présence simultanéc de tous les stades larvaires dans les prélèvements d'automne et d'hiver est due à la coexistence de deux cohortes, par exemple (1968) et (1969) dans les prélèvements d'automne 1969. Des nymphes $N_{3}$ apparaissent en avril et la période de vol s'étale de mai à septembre. Il n'est pas impossible que certains individus éclos précocement en août rattrapent ceux de la génération précédente el parviennent à accomplir leur cycle en un an (Décamps 1967). Toutefois, la longueur habituelle du cycle nous paraît être ici de deux ans comme l'indique la figure $3 \mathrm{~A}$.

A $1590 \mathrm{~m}$ d'altitude (ruisseau d'Artigusse), le cycle vital dure également deux ans (fig. $3 \mathrm{C}$ ). Comme à la station précédente, on observe un étalement des éclosions d'août à décembre, un ralentissement de la croissance en été, et un envol précoce des adultes en avril. Par contre, dans les prélèvements faits en Artigusse, on observe la présence constante de stades V, absents en Espiaube pendant la plus grande partic de l'été. Cette différence tient à la proximité des sources en Artigusse. En effet, les températures d'été étant plus basses, la cohorte âgée demeure plus longtemps au stade $V$ qu'en Espiaube; inversement, les températures d'hiver étznt plus élevées qu'en Espiaube, la jeune cohorte parvient plus rapidement au s!ade $V$.

A $1940 \mathrm{~m}$ d'altitude (ruisseau affluent d'Estaragne) le cycle vital dure trois ans (fig. $3 \mathrm{~B})$. Les œufs déposés en juillet-août n'éclosent qu'à partir de l'hiver, en novembre et décembre, jus- 
qu'en juin. L'étalement des éclosions, considérable, peut donc atteindre dix mois. La croissance a lieu principalement en été et au début de l'automne; elle s'arrête ou se ralentit pendant les autres périodes de l'année. On constate, comme en Artigusse, la présence constante de stades $\mathrm{V}$ dans les prélèvements. La nymphose est également allongée puisque, débutant en automne, elle ne s'achève qu'en mai - juin de l'année suivante, avec l'envol des adultes. Au total, le développement est arrêté en hiver à trois périodes du cycle vital : (1) au stade œuf, (2) vers le milieu du cycle, (3) au stade $V$ âgé ou nymphe $N_{1}$.

\section{3. - Cycle vital des cinq espèces de Micrasema dans le Nistos à $550 \mathrm{~m}$.}

Les cinq espèces pyrénéennes de Micrasema coexistent dans le Nistos à $550 \mathrm{~m}$. Elles accomplissent toutes leur cycle vital en 111 an (fig. 4).

(1) On note, chez Micrasema morosum, la présence simultanée de tous les stades larvaires dans les relevés à partir du quatrième mois. Cette dispersion traduit un étalement des éclosions plus important que pour les autres espèces.

(2) Les périodes de vol des adultes ne sont que peu ou pas décalées. Pour les larves, la plus grande partie de la croissance s'effectue entre les stades IV et $V$. A cette époque apparaissent les plus grands décalages dans le temps. $M$. morosum passe du stade IV au stade V, en moyenne, entre octobre et mars. Les autres espèces, à éclosions moins étalées, passent plus rapidement du stade IV au stade $\mathrm{V}$ : entre novembre et février pour $M$. longulum, entre janvier et mars pour $M$. difficile et $M$. minimum, entre mars et juin pour $M$. moestum.

(3) Sur les trois espèces récoltées également à d'autres stations, $M$. morosum présente des cycles différents (fig. 3 ); $M$. difficile et $M$. moestum présentent des cycles de même longueur avec de simples décalages dans les périodes de vol (fig. 4,5 et 6 ).

\section{4. $-\rightarrow$ Conclusion.}

Micrasema morosum s'oppose aux autres espèces par la variabilité de son cycle vital (fig. 3). Selon l'altitude, le développement de cette espèce dure en effet 1,2 ou 3 ans. Les autres espèces ont un cycle annuel. En particulier, le cycle vital de $M$. difficile est semblable à $470 \mathrm{~m}$ et $1590 \mathrm{~m}$. Notons que si cette espèce présente une certaine amplitude altitudinale, elle ne forme des populations abondantes qu'en des stations situées non loin des sources, vers $1500 \mathrm{~m}$ d'altitude. $M$. morosum, au cycle plus variable, se développe à différentes altitudes, sous des climats hydrologiques dissemblables; elle forme des populations prospères en moyenne et 
en haute altitude. Ceci confirme la relation déjà signalée (Décamps 1967) entre l'aptitude à coloniser divers milieux et la variabilité du cycle chez les Trichoptères de montagne.

A $1940 \mathrm{~m}$, on observe un allongement des stades oeuf et nymphe pour Micrasema morosum. L'allongement plus ou moins important du stade œuf entraîne un étalement des éclosions sur dix mois. Quant aux nymphes formées avant l'hiver, leur envol ne peut survenir qu'à la fonte des neiges qui en hiver recouvrent la station. En haute montagne, ces caractères paraissent communs à de nombreux insectes aquatiques, dont les œufs ou les nymphes apparaissent à la fin de l'été et ne peuvent achever leur développement avant l'hiver.

Les espèces présentes dans le Nistos à $550 \mathrm{~m}$, tout en appartenant au même genre, n'ont des périodes de vol que peu ou pas décalées. Leur mode d'alimentation est identique; elles se développent côte à côte dans les mousses sans qu'il soit possible de caractériser un microhabitat distinguant l'une ou l'autre de ces espèces. Ceci peut surprendre puisque ces espèces sont susceptibles d'entrer en compétition. Il convient ici de considérer la période la plus importante du cycle à cet égard, - celle séparant les deux dcrniers stades. Ainsi que nous l'avons vu, des différences apparaissent alors entre les espèces. L'identité des périodes de vol peut donc masquer des décalages dans les époques de plus forte croissance. Elle n'indique pas nécessairement une compétition importante aux stades larvaires.

\section{5. - CROISSANCE ET NUTRITION}

Pour chaque cohorte, l'accroissement du poids moyen, tel qu'il est indiqué par les courbes des figures 3 à 6 semble suivre une loi exponentielle. Cette hypothèse étant retenue, les logarithmes des poids moyens ont été placés par rapport au temps dans les figures 7 et 8 , et les droites moyennes tracées à vue. Cette représentation semi-logarithmique conduit aux conclusions suivantes:

(1) Pour une même cohorte, le taux de croissance est relativement constant à basse altitude. C'est le cas des cinq espèces vivant dans le Nistos à $550 \mathrm{~m}$ ( $f(g .7 \mathrm{~A}$ ). Aux altitudes plus élevées, des périodes de croissance nettement ralentic apparaissent dans une même cohorte : par exemple $M$. morosum à $1360 \mathrm{~m}$, à $1590 \mathrm{~m}$ et à $1940 \mathrm{~m}$ ( $f i g .7 \mathrm{~B}), M$. difficile à $1590 \mathrm{~m}$ (fig. 8). Ces cassures des taux de croissance coïncident avec la période hivernale à $1940 \mathrm{~m}$ et $1590 \mathrm{~m}$, avec les crues de fonte des neiges à $1360 \mathrm{~m}$. En cette dernière station, l'entraînement par dérive affecte les stades $\mathrm{V}$ plus que les stades jeunes. La cassure observée ici résulte plutôt de ce phénomène que d'un ralentissement de la croissance. 

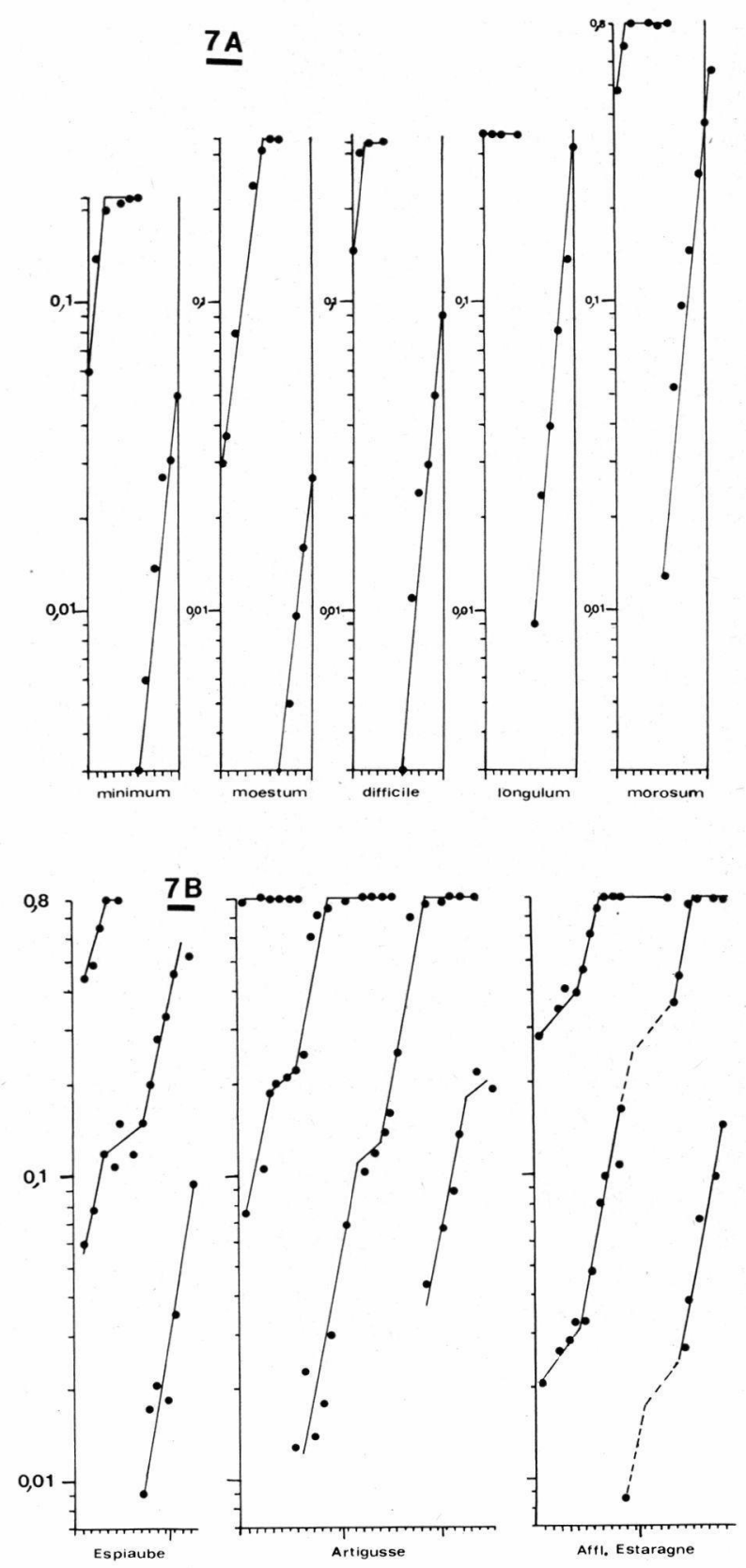

FIG. 7. - Croissance du poids moyen ( $\mathrm{mg}$ ) en ordonnée en fonction du temps (mois) en abscisse. $7 \mathrm{~A}$ : espèces vivant dans la rivière Le Nistos à $550 \mathrm{~m}$ d'altitude. $7 \mathrm{~B}$ : Micrasema morosum à diverses altitudes : Espiaube $(1360 \mathrm{~m})$, Artigusse $(1590 \mathrm{~m})$, Affl. d'Estaragne $(1940 \mathrm{~m})$. 


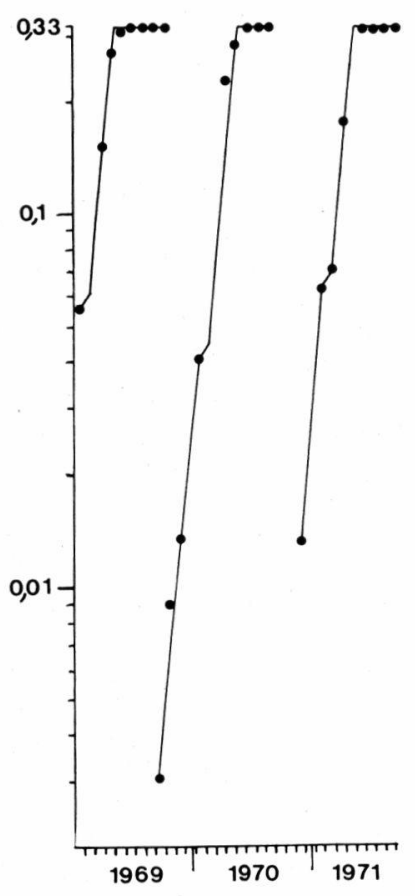

FIG. 8. - Croissance du poids moyen de Micrasema difficile à $1590 \mathrm{~m}$ d'altitude (ruisseau d'Artigusse).

(2) Dans une même station, les taux de croissance sont semblables d'une cohorte à la suivante aux périodes homologues de la vie larvaire. C'est notamment le cas de $M$. morosum à $1940 \mathrm{~m}$, à $1590 \mathrm{~m}$ (fig. $7 \mathrm{~B})$ et de $M$. difficile à $1590 \mathrm{~m}$ (fig. 8).

(3) Les taux de croissance les plus élevés apparaissent aux stations basses, chez des populations à cycle annuel. L'allongement des cycles vitaux de $\boldsymbol{M}$. morosum en haute altitude résulte donc à la fois de la présence de taux de croissance moindres entrecoupés de périodes à croissance nettement ralentie (fig. $7 \mathrm{~A}$ et $\mathrm{B}$ ).

A partir de la figure 8 , trois taux de croissance successifs ont été évalués pour une même cohorte (tableau 1$)$. Le premier $(R \simeq 0,8)$ conduit la larve de la sortie de l'œuf au $3^{\mathrm{e}}$ ou $4^{\mathrm{e}}$ mois. Le second $(0,10<\mathrm{R}<0,15)$ correspond à la période de fonte des neiges et dure environ un mois. Le troisième $(\mathrm{R} \simeq 0,8)$ conduit la larve au $7^{\mathrm{e}}$ mois au poids moyen du stade $\mathrm{V}$. A partir du $7^{\mathrm{e}}$ mois, la croissance moyenne est terminée : la nymphose commence. 
Tableau 1. - Evolution des cohortes de Micrasema difficile au ruisseau d'Artigusse $(1590 \mathrm{~m})$ : croissance du poids sec moyen individuel et diminution des nombres évalués à partir des figures 8 et $10 \mathrm{~B}$.

Cohorte (1968)

CoHorte (1969)

Cohonte (1970)

\begin{tabular}{|c|c|c|c|c|c|}
\hline \multicolumn{6}{|c|}{ Croissance $d u$ poids sec moyen individuel : $P_{t}=P_{0} e^{R t}$} \\
\hline $0-4$ mois & $\begin{array}{l}\mathrm{P}_{\mathrm{o}}=0,003 \\
\mathrm{R}=?\end{array}$ & $0-3$ mois & $\begin{array}{l}P_{n}=0,003 \\
R \simeq 0,80\end{array}$ & $0-4$ mois & $\begin{array}{l}\mathrm{P}_{\mathrm{o}}=0,003 \\
\mathrm{R} \simeq 0,80\end{array}$ \\
\hline 4-5 mois & $\begin{array}{l}\mathrm{P}_{\circ}=0,056 \\
\mathrm{R} \simeq 0,10\end{array}$ & 3-4 mois & $\begin{array}{l}\mathrm{P}_{\mathrm{o}}=0,036 \\
\mathrm{R} \simeq 0,15\end{array}$ & 4-5 mois & $\begin{array}{l}P_{0}=0,064 \\
R \simeq 0,12\end{array}$ \\
\hline $5-7 \mathrm{mo}$ & $\begin{array}{l}P_{\circ}=0,062 \\
R^{\circ} \simeq 0,80\end{array}$ & 4-7 mois & $\begin{array}{l}\mathrm{P}_{\circ}=0,042 \\
\mathrm{R} \simeq 0,80\end{array}$ & $5-7$ mois & $\begin{array}{l}\mathrm{P}_{\mathrm{o}}=0,072 \\
\mathrm{R} \simeq 0,80\end{array}$ \\
\hline
\end{tabular}

Diminution des nombres : $\mathrm{N}_{\mathrm{r}}=\mathrm{N}_{\mathrm{o}} \mathrm{e}^{-\mathrm{Zt}_{\mathrm{t}}}$

\begin{tabular}{|c|c|c|c|c|c|}
\hline & & $0-1$ mois & $\begin{array}{l}N_{\mathrm{o}}=18860 \\
\mathrm{Z} \simeq 0,85\end{array}$ & $2-8$ mois & $\begin{array}{l}N_{n}=25300 \\
Z \simeq 0,50\end{array}$ \\
\hline ?-8 mois & $z \simeq 0,22$ & 1-7 mois & $\begin{array}{l}\mathrm{N}_{\mathrm{o}}=8100 \\
\mathrm{Z} \simeq 0,20\end{array}$ & 8-12 mois & $\begin{array}{l}\mathrm{N}_{\mathrm{o}}=9000 \\
\mathrm{Z} \simeq 0,15\end{array}$ \\
\hline-12 mois & $\begin{array}{l}N_{\circ}=2600 \\
Z \simeq 1,45\end{array}$ & 7-12 mois & $\begin{array}{l}N_{o}=2500 \\
Z \simeq 1,35\end{array}$ & $0-2$ mois & $\begin{array}{l}N_{n}=3600 \\
Z^{2} \simeq 1,45\end{array}$ \\
\hline
\end{tabular}

Du point de vue de la nutrition, trois types d'éléments forment la totalité des contenus stomacaux des larves de Micrasema :

- des fragments de mousses (tiges et feuilles),

- des algues périphytiques,

— divers débris indifférenciables.

Les observations failes sur les différents échantillons montrent qu'un pourcentage élevé de larves présente un tube digestif plein quelle que soit la saison. Les algues périphytiques forment l'essentiel de l'alimentation des jeunes stades; mais à partir des stades III et IV, les tiges et les feuilles de mousses dominent largement. Des tubes diges'ifs vides n'ont été observés que chez des individus venant d'accomplir une mue larvaire ou chez des larves en fin de stade $\mathrm{V}$, pourvues d'abondantes réserves de graisses, avant la nymphose. Au total, les larves de Micrasema se nourrissent pendant toute la durée de leur cycle avec quelques arrêts aux périodes de mue; elles ne présentent pas d'arrêt saisonnier.

Peu de différences ont été décelées entre les espèces de Micrasema : les tiges et les feuilles de mousses forment la base de leur 
alimentation. Seules, les larves de $M$. moestum du Volp (à $430 \mathrm{~m}$ ) consomment une quantité relativement plus importante d'algues vertes.

\section{6. - FÉCONDITÉ ET ÉVOLUTION DES NOMBRES}

Sauf pour $M$. longulum (accord avec loi de Poisson non contredite : $\mathrm{P}>0,05$ ) et pour $M$. difficile (plus de 30 pontes dénombrées), les limites de confiance ont été calculées après transformation logarithmique et les coefficients obtenus appliqués aux moyennes arithmétiques (voir Elliott et Décamps 1973).

Pour chaque espèce, le nombre d'œufs par ponte est indiqué dans le tableau 2.

Tableau 2. - Nombre d'œufs par ponte des Micrasema pyrénéennes.

\begin{tabular}{lcc}
\hline & $\begin{array}{c}\text { Moyenne arithmétique } \\
\text { et } 95 \% \text { des limites } \\
\text { de confiance }\end{array}$ & $\begin{array}{c}\text { Intervalle } \\
\text { de confiance }\end{array}$ \\
\hline M. morosum & $137 \stackrel{\div}{\div} 0,83$ & 113 à 165 \\
M. longulum & $127 \pm 12$ & 115 à 139 \\
$M$. moestum & $124 \stackrel{\times}{\div} 1,2$ & 103 à 149 \\
$M$. difficile & $92 \pm 6$ & 86 à 98 \\
$M$. minimum & $70 \stackrel{\div}{\div} 0,85$ & 59 à 82 \\
\hline
\end{tabular}

Les deux espèces les plus petites ( $M$. difficile et minimum) ont un nombre d'œufs par ponte nettement inférieur aux autres espèces. Ces deux espèces forment les populations les plus abondantes observées dans cette étude, la première au ruisseau d'Artigusse à $1590 \mathrm{~m}$, la seconde à la rivière le Nistos à $550 \mathrm{~m}$.

L'évolution du nombre de larves d'une même cohorte reflète la mortalité et les mouvements des individus aux limites de l'aire échantillonnée. Dans le cas des Micrasema, cette évolution se présente différemment selon la densité de la couverture de mousses sur le fond du cours d'eau.

(1) Dans les stations où les mousses ne recouvrent qu'une faible proportion du fond, les populations de basse et haute altitude doivent être distinguées.

En basse altilude (fig. 9 A et $\mathrm{C}$ ), entre juillet (éclosion des œufs) et décembre, on constate une tendance générale à l'augmentation du nombre des larves, en dépit de fluctuations probablement dues 

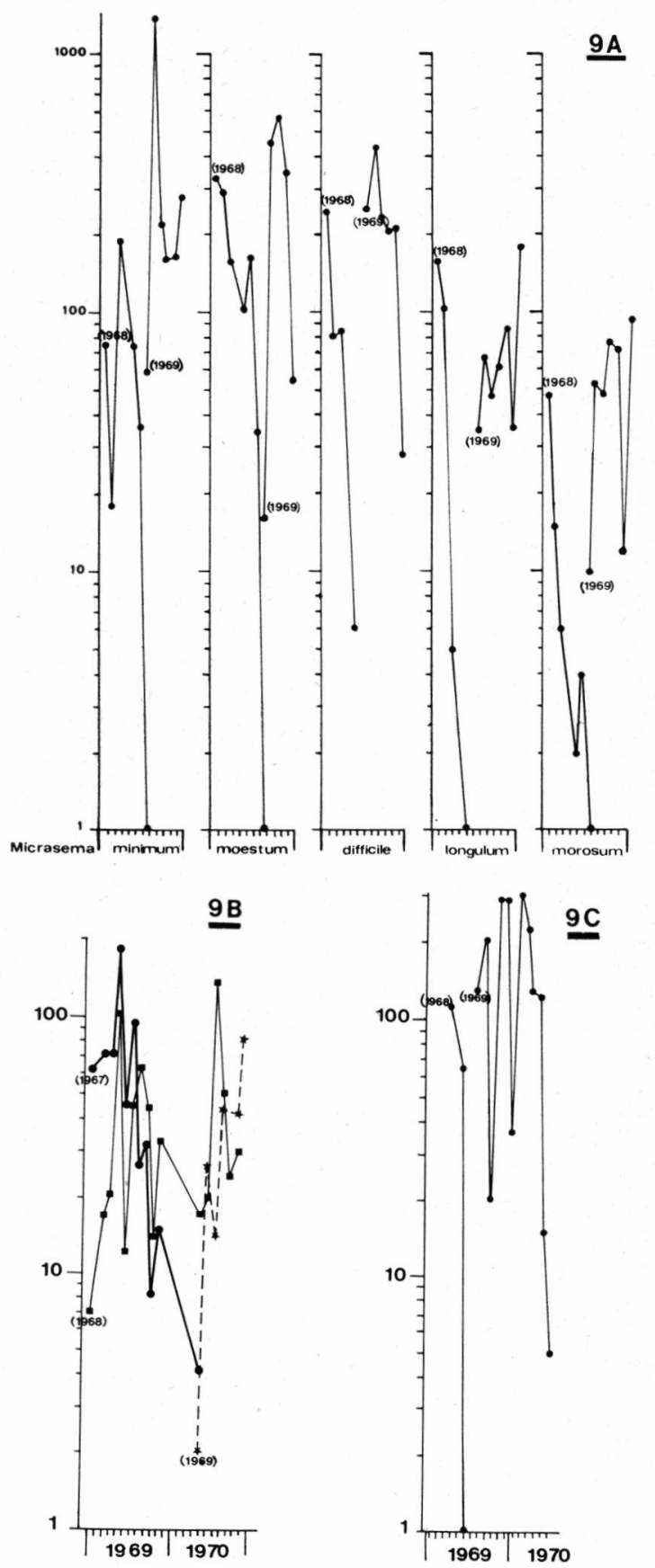

Fig. 9. - Evolution des nombres pendant le cycle vital de diverses populations de Micrasema en des stations faiblement recouvertes de mousses : rivière Le Nistos à $550 \mathrm{~m}(9 \mathrm{~A})$; ruisseau affluent d'Estaragne à $1940 \mathrm{~m}$ $(9 \mathrm{~B})$; rivière Le Volp à $430 \mathrm{~m}(9 \mathrm{C})$. 


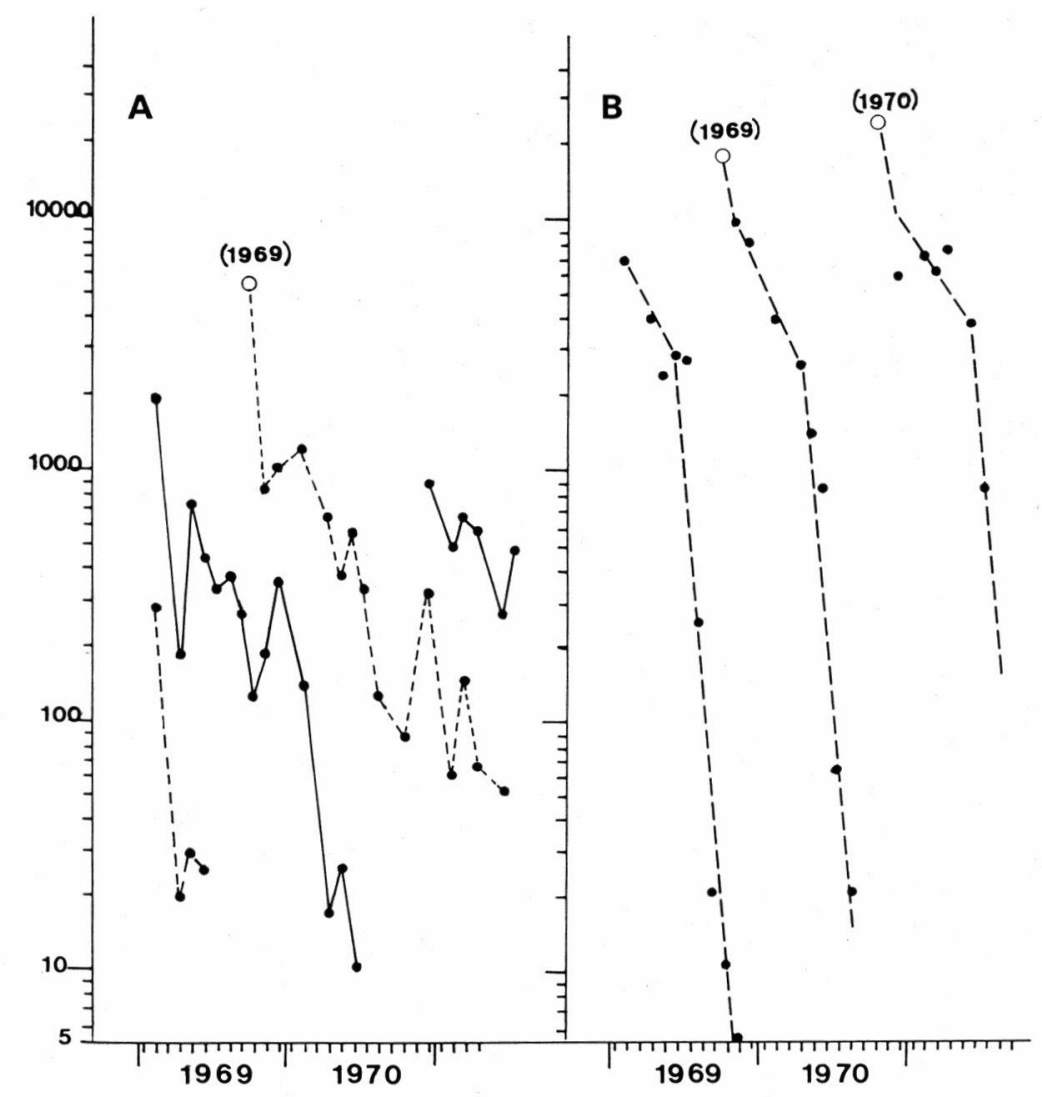

Fig. 10. - Evolution des nombres pendant le cycle vital de Micrasema morosum et $M$. difficile en une station fortement recouverte de mousses : le ruisseau d'Artigusse à $1590 \mathrm{~m}$ (les cercles blanes correspondent aux évaluations du nombre d'œufs $/ \mathrm{m}^{2}$ : voir tableau 3 ).

à l'échantillonnage. Cette augmentation doit être attribuée à une concentration dans les mousses d'individus précédemment éclos sous les pierres voisines. Entre janvier et juin, une diminution importante et souvent continue des nombres reflète un déflacement des larves avant la nymphose puis la métamorphose : cohortes (1968) dans les figures 9 A et 9 C. A cette époque, de nombreuses larves vont effectuer leur nymphose sous les pierres ou sont entraînées par dérive.

En haute altitude ( $f i g .9 \mathrm{~B}$ ), l'étalement des éclosions conduit à une augmentation continue des nombres pendant les premiers mois : cohorte (1969). Une concentration importante dans les 
mousses se produit aux stades IV et $\mathrm{V}$ : pics des cohortes (1967) et (1968) sur la figure $9 \mathrm{~B}$; elle précède, comme en basse altitude, une diminution continue du nombre des larves : cohorte (1967).

Dans ces stations existe une dérive non négligeable des différents stades larvaires de Micrasema.

(2) Dans la station d'Artigusse, les mousses recouvrent une forte proportion du fond $(\S 2)$. Les deux populations de cette station Micrasema morosum et $M$. difficile - diminuent plus régulièrement que les populations des autres stations (fig. 10). On n'observe pas de concen!ration de larves en provenance des pierres : la quasitotalité du développement larvaire se déroule dans les mousses. Seules, certaines larves du stade $\mathrm{V}$ quittent les mousses pour $\mathrm{s}$ ? fixer sous les pierres lors de la nymphose. Cette période exceptée, la dérive étant par ailleurs très faible ou nulle, la diminution des nombres peut être considérée comme une indication du taux de mortalité.

Les deux cas doivent donc être distingués dans une étude de la dynamique des populations de Micrasema. Dans le premier cas, les larves n'accomplissent qu'une partie de leur développement dans les mousses; un échantillonnage des mousses seules est insuffisant pour analyser l'évolution démographique réelle de la population. Par contre, dans le second cas, lorsque les mousses recouvrent une forte proportion du fond, la présence continue des larves, depuis l'éclosion jusqu'à la nymphose, permet une évaluation de la production à partir de l'échantillonnage des mousses.

Les paramètres de la diminution des nombres sont présentés (tableau 1) pour $M$. difficile au ruisseau d'Artigusse $(1590 \mathrm{~m})$. Ces nombres ont été évalués à partir de la figure $10 \mathrm{~B}$. Pour chaque cohorte, trois périodes peuvent être distinguées; au cours de chacune de ces périodes les taux de mortalité $Z$ (comprenant les pertes par déplacement et nymphose) sont constants. A la différence des taux de croissance $R$ du même tableau 1 , les valeurs de $Z$ varient d'une cohorte à la suivante pour des périodes homologies. On peut toutefois résumer l'évolution des populations de $\boldsymbol{M}$. difficile à partir du tableau 1 et de la figure $10 \mathrm{~B}$. A partir de l'éclosion, les nombres diminuent rapidement pendant un ou deux mois. Suit une longue période de six mois au cours de laquelle la diminution des nombres est faible. Pendant ces deux premières périodes, les valeurs de $Z$ sont voisines du taux de mortalité. Enfin, la diminution des nombres s'accélère au cours des cinq ou six derniers mois. A cette période, les valeurs de $Z(1,45-1,35-1,45)$ reflètent à la fois la mortalité et la transformation des larves en nymphes. 


\section{7. - BIOMASSES ET PRODUCTION}

\section{1. - Estimation de la surface d'un relevé au ruisseau d'Artigusse.}

Pour le ruisseau d'Artigusse à $1590 \mathrm{~m}$ d'altitude, le poids sec moyen de mousses a été évalué à $325 \mathrm{~g} / \mathrm{m}^{2}$ au mois de juin (Dawson 1973). Les sondages réalisés à trois autres moments de l'année permettent de considérer cette valeur comme moyenne pour l'ensemble de la période étudiée : de janvier 1969 à juillet 1971. Sur cette base, il est possible d'estimer approximativement la surface des relevés faits au ruisseau d'Artigusse. En effet, les volumes prélevés (900 $\mathrm{cm}^{3}$ de mousses), ayant un poids sec moyen égal à $65 \pm 9 \mathrm{~g}(\mathrm{P}>0,95)$, chaque relevé correspond, en moyenne, à une surface égale à :

$$
65 \div 325=0,2 \mathrm{~m}^{2} .
$$

Ce raisonnement ne sera retenu, avec quelque vraisemblance, que pour le ruisseau d'Artigusse à $1590 \mathrm{~m}$, station où la couverture de mousses présente une relative constance dans l'espace et dans le temps. A cette station, le nombre de larves par relevé doit donc être multiplié par 5 pour obtenir le nombre de larves par $\mathrm{m}^{2}$.

\section{2. - Biomasses des populations de Micrasema morosum aux différentes altitudes.}

L'évolution des biomasses de Micrasema norosum a été considérée aux différentes altitudes (fig. 11). Pour chaque cohorte, les plus fortes biomasses sont atleintes lorsque les stades $\mathrm{V}$ sont les plus nombreux, c'est-à-dire avant le début de la nymphose. Cette période de biomasse maximale varie selon les stations, puisque, située en hiver de 550 à $1590 \mathrm{~m}$ d'altitude, elle se présente au cours de l'été à $1940 \mathrm{~m}$. Les plus fortes périodes de croissance se produisent en effet en automne aux trois premières stations, en été à la dernière (fig. 11). Mais ceci n'a pratiquement pas d'influence sur la période de vol : le décalage entre l'époque de biomasse maximale et le début de la période de vol, faible en basse et moyenne altitude, augmente à la station la plus haute par suite de l'allongement du stade nymphal.

Les graphiques de la figure 11 concernent des nombres par volume de mousses $\left(900 \mathrm{~cm}^{3}\right.$ par relevé). Sur cette base, les biomasses de Micrasema morosum sont les plus importantes à $1940 \mathrm{~m}$, ce qui est en accord avec le caractère montagnard de cette espèce. Mais il n'en est plus de même, si au lieu de volumes de mousses, la comparaison porte sur des surfaces, - le $\mathrm{m}^{2}$ par 

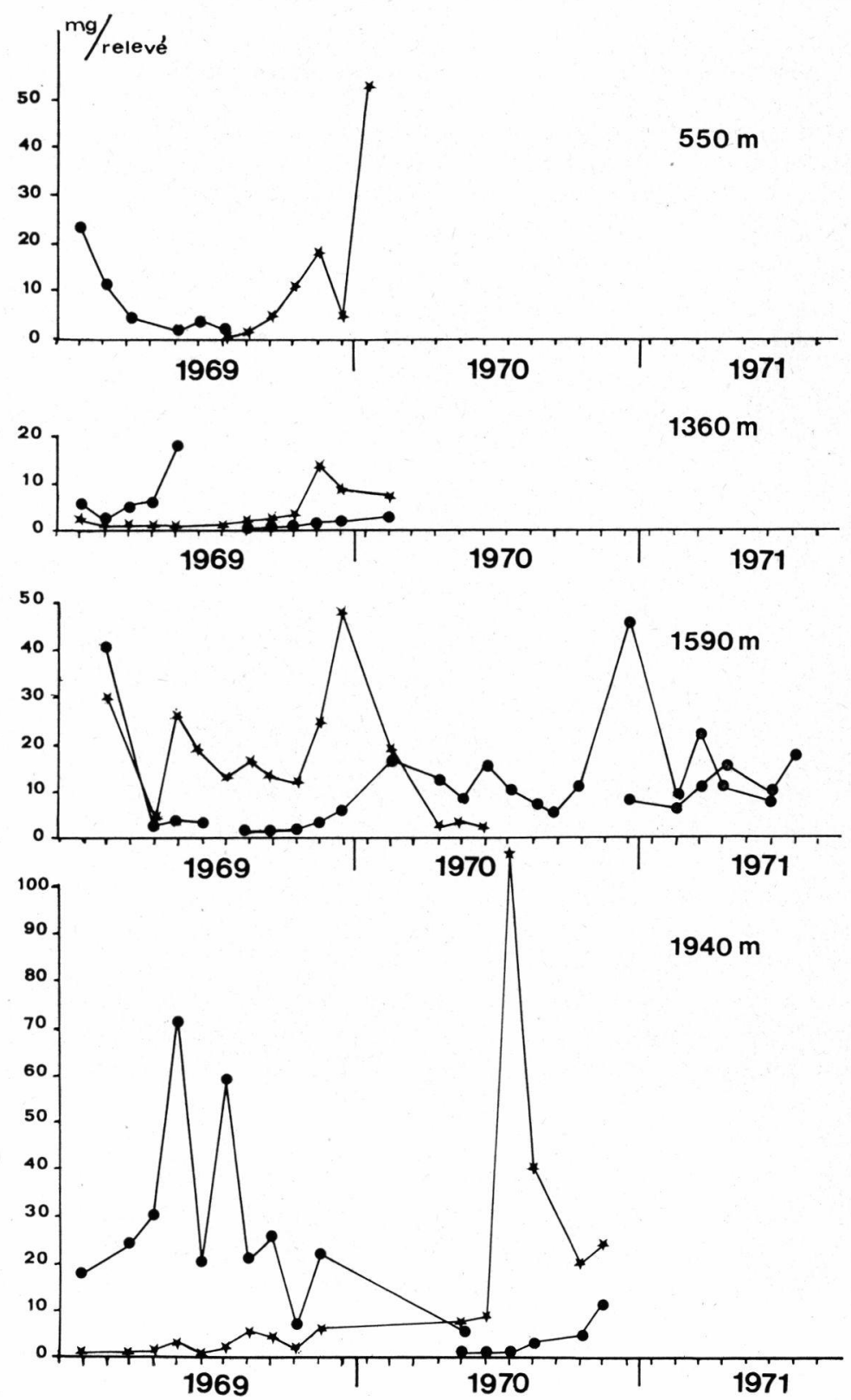

Fig. 11. - Evolution des biomasses pendant le cycle vital de Micrasema morosum à différentes altitudes : Le Nistos $(550 \mathrm{~m})$, Espiaube $(1360 \mathrm{~m})$, Artigusse $(1590 \mathrm{~m})$ et l'affluent d'Estaragne $(1940 \mathrm{~m})$. 


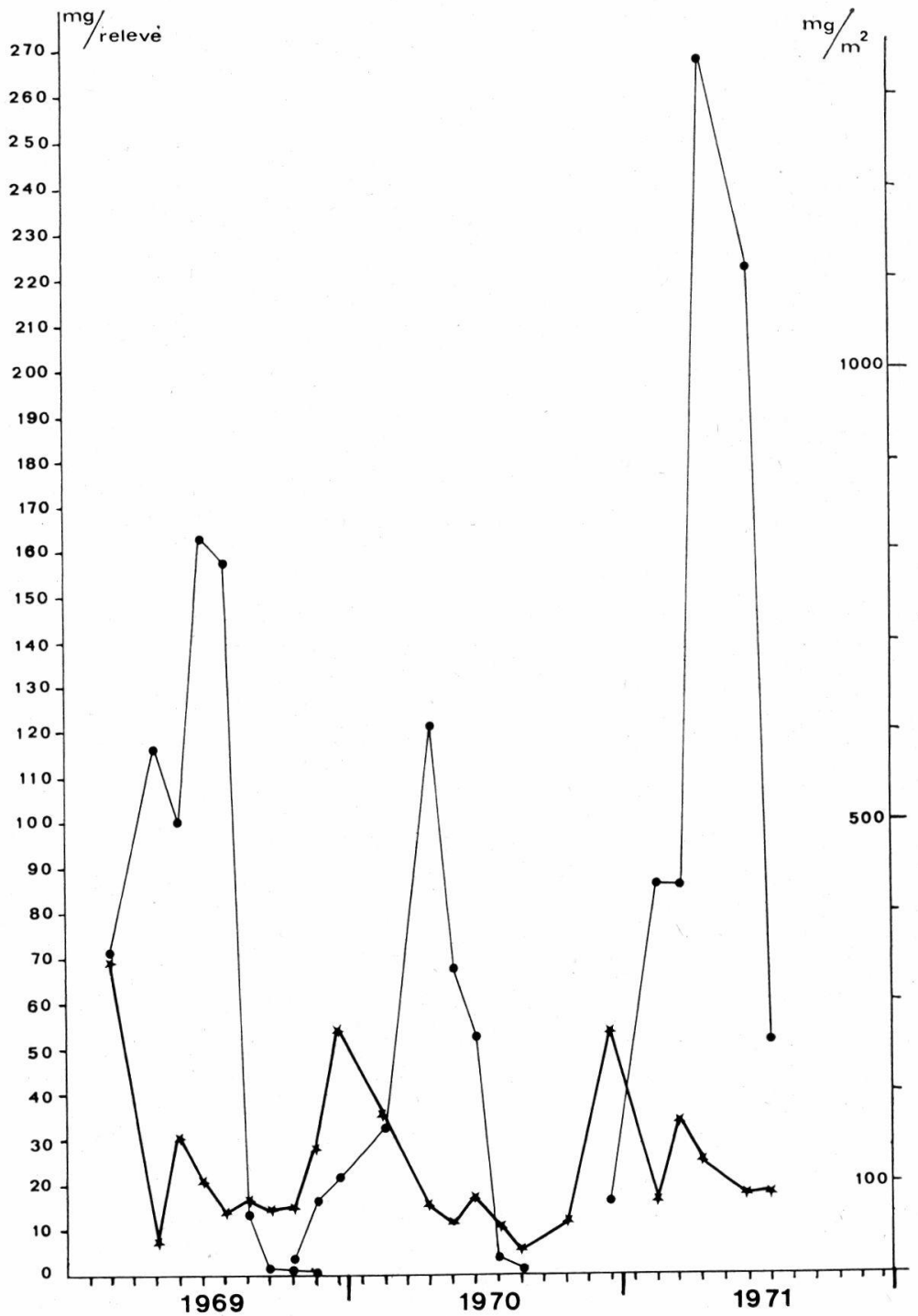

Fig, 12. - Evolution des biomasses pendant le cycle vital de Micrasema difficile (trait fin) et $M$. morosum (trait épais) au ruisseau d'Artigusse (1590 m). 


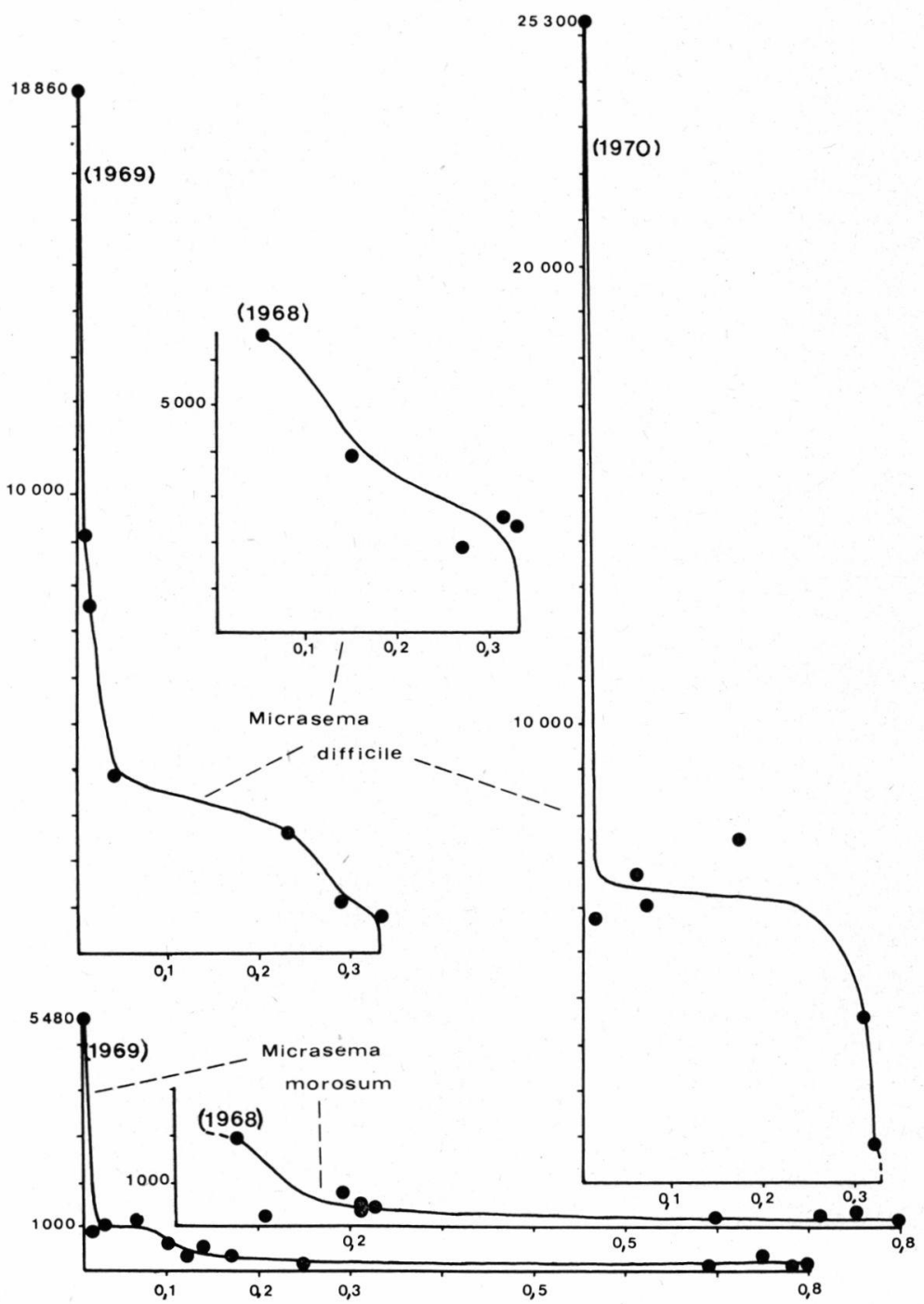

Fig. 13. - Courbes de croissance - survie de plusieurs cohortes de Micrasema difficile et $M$. morosum au ruisseau d'Artigusse (1590 m).

exemple. En effet, pour une évaluation au $\mathrm{m}^{2}$, les nombres doivent être multipliés par 5 au ruisseau d'Artigusse à $1590 \mathrm{~m}$ (voir $\S$ précédent). A titre de comparaison, nous pouvons estimer que les nombres doivent être multipliés par 1,4 à $1940 \mathrm{~m}$ (où le poids moyen de mousses atteint $92 \mathrm{~g} / \mathrm{m}^{2}$ (Dawson 1973) : $65 \div 92 \simeq 0,7$ ); 
ils doivent être multipliés par un nombre égal ou inférieur à 1 pour les trois stations situées de $430 \mathrm{~m}$ à $1360 \mathrm{~m}$ d'altitude. Il s'ensuit que les plus fortes biomasses par unité de surface sont atteintes à la station d'Artigusse $(1590 \mathrm{~m})$. Moins que la quantité de nourriture disponible, le nombre d'abris offerts par une plus grande quantité de mousses au $\mathrm{m}^{2}$ est probablement à l'origine de ces différences.

\section{3. - Biomasses de Micrasema morosum et $M$. difficile au ruisseau d'Artigusse.}

L'évolution des biomasses de ces deux espèces a été représentée sur une double ordonnée : en $\mathrm{mg} /$ relevé et en $\mathrm{mg} / \mathrm{m}^{2}$ (fig. 12). Pour $M$. morosum, dont le cycle s'étend sur deux ans, les biomasses totales (sommes des biomasses des différentes cohortes présentes dans le même relevé) ont été utilisées. Pour $M$. difficile, dont le cycle s'étend sur un an, les biomasses indiquées concernent trois cohortes successives. Un décalage apparaît clairement entre les deux espèces pour les périodes de croissance el de biomasse maximale. Cette réduction de la compétition, jointe à l'abondance de mousses, peut expliquer les fortes densités atteintes par $\boldsymbol{M}$. morosum et difficile en cette station.

\section{4. - Estimation du nombre maximal d'œufs pondus par M. moro- sum et $M$. difficile au ruisseau d'Artigusse.}

Le nombre d'œufs à l'origine d'une cohorte a été évalué à partir des nymphes $\mathrm{N}_{3}$ de la cohorte précédente. Le relevé le plus riche en nymphes a été retenu pour cette estimation (tableau 3).

Tableau 3. - Calcul du nombre maximal d'œufs $/ \mathrm{m}^{2}$ pondus au ruisseau d'Artigusse à $1590 \mathrm{~m}$ par Micrasema difficile et $M$. morosum (" voir tableau 2).

\begin{tabular}{|c|c|c|c|c|c|}
\hline $\begin{array}{l}\text { Nombre } \\
\text { de } \\
\text { nymphes/ } \\
\text { relevé } \\
\text { (N) }\end{array}$ & $\begin{array}{c}\text { Nombre } \\
\text { de } \\
\text { nymphes } \\
q / \text { relevé } \\
\left(n_{1}\right)\end{array}$ & $\begin{array}{c}\text { Nombre } \\
\text { de } \\
\text { nymphes } \\
q / \mathrm{m}^{2} \\
\left(\mathrm{n}_{\mathrm{q}}=\mathrm{n}_{1} \times 5\right)\end{array}$ & $\begin{array}{l}\text { Nombre } \\
\text { moyen } \\
\text { d'œufs/m² }\end{array}$ & $\begin{array}{c}\text { Limite } \\
\text { inférieure }\end{array}$ & $\begin{array}{l}\text { Limite } \\
\text { supérieure }\end{array}$ \\
\hline
\end{tabular}

\begin{tabular}{|c|c|c|c|c|c|c|}
\hline M. difficile & & & & $\left(\mathrm{n}_{2} \times 92\right)^{*}$ & $(\mathrm{n}, \times 86)^{*}$ & $(n, \times 98)^{*}$ \\
\hline-1969 & 80 & 41 & 205 & 18860 & 17630 & 20090 \\
\hline-1970 & 115 & 55 & 275 & 25300 & 23650 & 26950 \\
\hline $\begin{array}{c}\text { M. morosum } \\
-1969\end{array}$ & 15 & 8 & 40 & $\begin{array}{c}\left(n_{2} \times 137\right)^{*} \\
5480\end{array}$ & $\begin{array}{c}\left(\mathrm{n}_{2} \times 113\right)^{\star} \\
4520\end{array}$ & $\begin{array}{c}\left(\mathrm{n}_{2} \times 165\right)^{*} \\
6600\end{array}$ \\
\hline
\end{tabular}


Pour Micrasema difficile, sur les 80 nymphes (cohorte 1968) du relevé d'août 1969 , on compte 41 \%, soit $41 \times 5=205$ nymphes $\mathrm{q} / \mathrm{m}^{2}$. En supposant que toutes ces femelles pondent, le nombre moyen d'œufs $/ \mathrm{m}^{2}$ de la cohorte 1969 s'élèvera à $205 \times 92$ $=18860$.

De même, sur les 115 nymphes (cohorte 1969) du relevé d'août 1970 , on compte 55 \%, soit $55 \times 5=275$ nymphes $\mathrm{q} / \mathrm{m}^{2}$. Le nombre moyen d'œufs $/ \mathrm{m}^{2}$ de la cohorte 1970 s'élèvera à $275 \times 92=25300$.

Tableau 4. - Calcul de la production de Micrasema difficile à partir des accroissements successifs de biomasse.

\begin{tabular}{|c|c|c|c|c|c|c|}
\hline Date & $\begin{array}{c}\text { Biomasse } \\
\text { moyenne } \\
\text { d'un } \\
\text { individu } \\
\text { mg } \\
\text { (a) }\end{array}$ & $\begin{array}{l}\text { Accrois- } \\
\text { sement } \\
\text { de } \\
\text { biomasse } \\
\text { d'un } \\
\text { individu } \\
\text { (b) }\end{array}$ & $\begin{array}{l}\text { Nombre } \\
\text { d'individus } \\
\text { par } \mathrm{m}^{2} \\
\text { (c) }\end{array}$ & $\begin{array}{l}\text { Nombre } \\
\text { moyen } \\
\text { à deux } \\
\text { intervalles } \\
\text { successifs } \\
\text { ind./m² } \\
\text { (d) }\end{array}$ & $\begin{array}{c}\underset{\mathrm{mg}}{\mathrm{P} / \mathrm{m}^{2}} \\
\text { (b) } \times(\mathrm{d})\end{array}$ & $\begin{array}{c}\mathrm{B} \\
\mathbf{m g} / \mathbf{m}^{2} \\
\text { (a) } \times(\mathrm{c})\end{array}$ \\
\hline \multicolumn{7}{|c|}{ Cohorte (1968) } \\
\hline $\begin{array}{l}2.69 \\
4 . \\
5 . \\
6 . \\
7 . \\
8 . \\
9 . \\
10 . \\
11 .\end{array}$ & $\begin{array}{l}0,056 \\
0,15 \\
\mathbf{0 , 2 7} \\
\mathbf{0 , 3 2} \\
\mathbf{0 , 3 3} \\
\mathbf{0 , 3 3} \\
\mathbf{0 , 3 3} \\
\mathbf{0 , 3 3} \\
0,33\end{array}$ & $\begin{array}{l}\overline{0,09} \\
0,12 \\
0,05 \\
0,01\end{array}$ & $\begin{array}{r}6455 \\
3830 \\
1815 \\
2560 \\
2405 \\
210 \\
20 \\
10 \\
5\end{array}$ & $\begin{array}{r}5142 \\
2822 \\
2187 \\
2482 \\
1307 \\
110 \\
15 \\
7\end{array}$ & $\begin{array}{r}46 \\
34 \\
11 \\
2 \\
\end{array}$ & 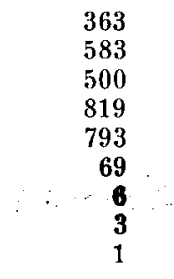 \\
\hline \multicolumn{7}{|c|}{ Cohorte (1969) } \\
\hline $\begin{array}{l}10.69 \\
11 . \\
12 . \\
2.70 \\
4 . \\
5 . \\
6 . \\
7 . \\
8 .\end{array}$ & $\begin{array}{l}\mathbf{0 , 0 0 3} \\
\mathbf{0 , 0 0 9} \\
0,014 \\
\mathbf{0 , 0 4 2} \\
\mathbf{0 , 2 3} \\
\mathbf{0 , 2 9} \\
\mathbf{0 , 3 3} \\
\mathbf{0 , 3 3} \\
\mathbf{0 , 3 3}\end{array}$ & $\begin{array}{l}\overline{0,006} \\
0,005 \\
0,028 \\
0,188 \\
0,06 \\
0,04 \\
\end{array}$ & $\begin{array}{r}18860 \\
9200 \\
7600 \\
3900 \\
2620 \\
1165 \\
800 \\
60 \\
\\
20\end{array}$ & $\begin{array}{r}-\overline{4030} \\
8400 \\
5750 \\
3260 \\
1892 \\
982 \\
430 \\
40 \\
P=1052\end{array}$ & $\begin{array}{r}84 \\
42 \\
161 \\
613 \\
113 \\
39\end{array}$ & $\begin{array}{r}56 \\
82 \\
106 \\
164 \\
602 \\
338 \\
264 \\
19 \\
6 \\
\max .=1,75\end{array}$ \\
\hline \multicolumn{7}{|c|}{ Cohorte (1970) } \\
\hline $\begin{array}{l}10.70 \\
12 . \\
2.71 \\
3 . \\
4 . \\
6 . \\
7 .\end{array}$ & $\begin{array}{l}0,003 \\
0,014 \\
0,064 \\
0,072 \\
0,178 \\
0,31 \\
0,32\end{array}$ & $\begin{array}{l}\overline{0,011} \\
0,050 \\
0,008 \\
0,106 \\
0,132 \\
0,010\end{array}$ & $\begin{array}{r}25300 \\
5575 \\
6 \quad 750 \\
5965 \\
7520 \\
3590 \\
805\end{array}$ & $\begin{aligned} 15487 \\
6212 \\
6357 \\
6737 \\
5555 \\
2197 \\
P=2010\end{aligned}$ & $\begin{array}{c}\overline{170} \\
310 \\
51 \\
714 \\
733 \\
22 \\
\mathrm{P} / \mathrm{B}\end{array}$ & $\begin{array}{c}75 \\
79 \\
432 \\
429 \\
1338 \\
1112 \\
257 \\
\max .=1,50\end{array}$ \\
\hline
\end{tabular}


Pour Micrasema morosum, sur les 15 nymphes (cohorte 1967) du relevé de février 1969 , on compte $8 \%$, soit $8 \times 5=40$ nymphes $q / \mathrm{m}^{2}$. Le nombre moyen d'œufs $/ \mathrm{m}^{2}$ de la cohorte 1969 s'élèvera à $40 \times 137=5480$.

Ces nombres supposent que loutes les nymphes $q$ récoltées dans les mousses déposent leurs oufs; ils sont donc surestimés. En contre-partie, il n'est pas tenu compte dans ces calculs des nymphes fixées aux pierres. Une certaine compensation peut donc être espérée.

\section{5. - Production de Micrasema difficile et de M. morosum au ruisseau d'Artigusse.}

Les productions ont été évaluées selon trois méthodes distinctes.

(1) La méthode graphique d'Allen (1951) a été appliquée à des courbes de croissance-survie tracées pour chaque cohorte (fig. 13). Les aires situées sous les courbes ont été évaluées à l'aide d'un planimètre. Cette méthode est l'équivalent graphique de la méthode suivante, la principale cause de différence tenant dans l'arrondi des courbes (fig. 13).

(2) Une méthode semblable à celle utilisée par Kajak et Ryback (1966), Andersson (1969), Giani et Laville (1973) a permis de calculer la production à partir de l'accroissement de biomasse moyenne d'un individu multiplié par le nombre moyen d'individus récoltés en deux échantillons successifs (tableaux 4 et 5 ).

(3) Dans la troisième méthode, la production a été évaluée par le produit du taux de croissance instantané et de la biomasse moyenne (Ricker 1946). A l'exemple d'Elliott (1973), les taux de croissance instantanés ont été estimés par les valeurs successives de $\mathbf{R}$ (tableau 1). La biomasse moyenne a été calculée à partir des équations de croissance du poids sec moyen individuel et de diminution des nombres (tableau 1). Les calculs figurent au tableau 6; cette évaluation n'a pu être faite que pour M. difficile.

Les résultats obtenus par ces trois méthodes sont regroupés tableau 7. La lecture de ce tableau conduit aux conclusions suivantes :

1. - Les trois méthodes donnent des résultats voisins pour chacune des cohortes considérées. Si les deux premières méthodes sont plus directement basées sur les nombres obtenus dans nos échantillonnages (malgré le tracé approché des courbes d'Allen), la troisième méthode s'appuie sur un modèle hypothétique de croissance $d u$ poids moyen individuel et de diminution des nombres (tableau 1) de Micrasema difficile. Les valeurs portées au tableau 7 permettent de considérer ce modèle comme vraisemblable. 
Tableau 5. - Calcul de la production de Micrasema morosum à partir des accroissements successifs de biomasse (cohorte 1969).

\begin{tabular}{|c|c|c|c|c|c|c|}
\hline Date & $\begin{array}{l}\text { Biomasse } \\
\text { moyenne } \\
\text { d'un } \\
\text { individu } \\
\text { mg } \\
\text { (a) }\end{array}$ & $\begin{array}{l}\text { Accrois- } \\
\text { sement } \\
\text { de } \\
\text { biomasse } \\
\text { d'un } \\
\text { individu } \\
\text { (b) }\end{array}$ & $\begin{array}{l}\text { Nombre } \\
\text { d'individus } \\
\text { par m² } \\
\text { (c) }\end{array}$ & $\begin{array}{l}\text { Nombre } \\
\text { moyen } \\
\text { à deux } \\
\text { intervalles } \\
\text { successifs } \\
\text { ind./m² } \\
\text { (d) }\end{array}$ & $\frac{\mathrm{P}}{\mathrm{mg} / \mathrm{m}^{2}}$ & $\frac{\mathrm{B}}{\mathrm{mg} / \mathrm{m}^{2}}$ \\
\hline $\begin{array}{l}10.69 \\
11 . \\
12 . \\
2.70 \\
4 . \\
5 . \\
6 . \\
7 . \\
8 . \\
10 . \\
12 . \\
2.71 \\
3 . \\
4 . \\
6 .\end{array}$ & $\begin{array}{l}0,009 \\
0,018 \\
0,03 \\
0,069 \\
0,1 \\
0,12 \\
0,14 \\
0,17 \\
0,25 \\
0,69 \\
0,75 \\
0,78 \\
0,8 \\
0,8 \\
0,8\end{array}$ & $\begin{array}{c}\overline{0} \\
0,009 \\
0,012 \\
0,039 \\
0,031 \\
0,020 \\
0,020 \\
0,030 \\
0,080 \\
0,440 \\
0,060 \\
0.030 \\
0,020\end{array}$ & $\begin{array}{r}5480 \\
850 \\
1000 \\
1195 \\
625 \\
350 \\
565 \\
315 \\
110 \\
85 \\
310 \\
60 \\
140 \\
65 \\
50\end{array}$ & $\begin{array}{r}3 \overline{165} \\
925 \\
1097 \\
910 \\
487 \\
457 \\
440 \\
212 \\
97 \\
197 \\
180 \\
100 \\
102 \\
57\end{array}$ & $\begin{array}{r}- \\
28 \\
11 \\
42 \\
28 \\
10 \\
9 \\
13 \\
17 \\
42 \\
12 \\
5 \\
2\end{array}$ & $\begin{array}{r}49 \\
15 \\
30 \\
82 \\
62 \\
42 \\
79 \\
53 \\
27 \\
58 \\
232 \\
46 \\
112 \\
52 \\
40\end{array}$ \\
\hline & & & & & $\overline{209}$ & $\begin{array}{l}P / B \max . \\
=0,90\end{array}$ \\
\hline
\end{tabular}

Tabieau 6. -- Evaluation de la production par le produit du taux de croissance instantané et de la biomasse moyenne : Micrasema difficile au ruisseau d'Artigusse.

\begin{tabular}{|c|c|c|c|c|c|c|}
\hline $\begin{array}{c}\text { Age } \\
(\text { mois })\end{array}$ & $\begin{array}{l}\text { Biomasse } \\
\text { d'un } \\
\text { individu } \\
\text { (mg) }\end{array}$ & $\begin{array}{c}\text { Nombre } \\
/ \mathrm{m}^{2}\end{array}$ & $\begin{array}{c}\text { Biomasse } \\
\text { totale } \\
\left(\mathrm{mg} / \mathrm{m}^{2}\right)\end{array}$ & $\begin{array}{l}\text { Biomasse } \\
\text { moyenne } \\
\left(\mathrm{mg} / \mathrm{m}^{2}\right)\end{array}$ & $\begin{array}{l}\text { Taux de } \\
\text { croissance }\end{array}$ & $\begin{array}{l}\text { Production } \\
\left(\mathrm{mg} / \mathrm{m}^{2}\right)\end{array}$ \\
\hline \multicolumn{7}{|c|}{ Cohorte (1969) } \\
\hline $\begin{array}{r}0 \\
1 \\
2 \\
3 \\
4 \\
5 \\
6 \\
7 \\
8 \\
9 \\
10 \\
11 \\
12\end{array}$ & $\begin{array}{l}0,003 \\
0,007 \\
0,015 \\
0,036 \\
0,042 \\
0,09 \\
0,20 \\
0,33 \\
0,33 \\
0,33 \\
0,33 \\
0,33 \\
0,33\end{array}$ & $\begin{array}{r}18860 \\
8100 \\
6600 \\
5400 \\
4500 \\
3600 \\
3000 \\
2500 \\
650 \\
160 \\
40 \\
10\end{array}$ & $\begin{array}{r}56,58 \\
56,70 \\
99,00 \\
194,40 \\
189,00 \\
324,00 \\
600,00 \\
825,00 \\
214,50 \\
52,80 \\
13,20 \\
3,30\end{array}$ & $\begin{array}{r}56,64 \\
77,85 \\
146,70 \\
191,70 \\
256,50 \\
462,00 \\
712,50 \\
519,75 \\
133,65 \\
33,00 \\
8,25\end{array}$ & $\begin{array}{l}0,8 \\
0,8 \\
0,8 \\
0,15 \\
0,8 \\
0,8 \\
0,8 \\
- \\
- \\
- \\
-\end{array}$ & $\begin{array}{r}45,3 \\
62,3 \\
117,4 \\
28,7 \\
205,2 \\
369,6 \\
570,0 \\
- \\
- \\
-\end{array}$ \\
\hline \multicolumn{7}{|c|}{ Cohorte (1970) } \\
\hline $\begin{array}{r}0 \\
1 \\
2 \\
3 \\
4 \\
5 \\
6 \\
7 \\
8 \\
9 \\
10 \\
11 \\
12\end{array}$ & $\begin{array}{l}0,003 \\
0,006 \\
0,014 \\
0,030 \\
0,064 \\
0,072 \\
0,155 \\
0,33 \\
0,33 \\
0,33 \\
0,33 \\
0,33 \\
0,33\end{array}$ & $\begin{array}{r}25300 \\
15000 \\
9000 \\
7600 \\
6600 \\
5700 \\
4800 \\
4150 \\
3600 \\
1400 \\
300 \\
75 \\
\\
20\end{array}$ & $\begin{array}{r}75,90 \\
90,00 \\
126,00 \\
228,00 \\
422,40 \\
410,40 \\
744,00 \\
1369,50 \\
1188,00 \\
462,00 \\
99,00 \\
24,75 \\
6,60\end{array}$ & $\begin{array}{r}82,9 \\
108,0 \\
177,0 \\
325,2 \\
416,4 \\
577,2 \\
1056,7 \\
1378,7 \\
825,0 \\
280,5 \\
61,8 \\
15,7\end{array}$ & $\begin{array}{c}0,8 \\
0,8 \\
0,8 \\
0,8 \\
0,12 \\
0,8 \\
0,8 \\
- \\
- \\
- \\
-\end{array}$ & $\begin{array}{r}66,3 \\
86,4 \\
141,6 \\
260,1 \\
50,0 \\
461,8 \\
845,4 \\
- \\
- \\
- \\
- \\
1911,6\end{array}$ \\
\hline
\end{tabular}


'Tableau 7. - Evaluation des production, biomasse maximale et rapport $P: B$ max. pour Micrasema difficile et M. morosum du ruissean d'Artigusse.

$\mathrm{P}\left(\mathrm{mg} / \mathrm{m}^{2}\right) \quad \mathrm{Bmax} . \quad \mathrm{P} / \mathrm{Bmax}$

(1) Wéthode graphique.

M. difficile :

cohorte 1969

cohorte 1970

1085

2025

625

1400

1,74

M. morosum :

cohorte 1969

230

210

1,09

(2) Calcul à partir des accroissements successifs de biomasse.

$\begin{array}{lccc}\begin{array}{l}M . \text { difficile } \\ \text { cohorte } 1969\end{array} & & & \\ \text { cohorte } 1970 & 1052 & 602 & 1,75 \\ \begin{array}{c}M . \text { morosum } \\ \text { cohorte } 1969\end{array} & 2010 & 1338 & 1,50 \\ & & & \\ \end{array}$

(3) Calcul à partir des taux de croissance instantanés.

M. difficile :

cohorte 1969

cohorte 1970

1398

1912

825

1369

1,69

1,40

2. - De nettes variations apparaissent entre les productions de deux cohortes successives d'une même espèce, de même qu'entre les productions des deux espèces de Micrasema présentes au ruisseau d'Artigusse. Cetle évolution dissemblable des populations de M. difficile et $M$. morosum est Iraduite par l'aspect des courbes de la figure 13. Même pour une station donnée, une valeur unique de production, obtenue une certaine année, ne peut servir à caractériser une espèce donnéc ou, a fortiori, des espèces appartenant à un genre donné. En ce sens, les exigences de précision systématique ne sont pas moindres dans les études de production que dans les autres études écologiques.

3. - Les productions et biomasses maximales des Micrasema du ruisseau d'Artigusse sont du même ordre de grandeur que celles obtenues par Pearson et Kramer (1972) pour un Trichoptère américain. Ces auteurs donnent en effet pour Oligophlebodes sigma des biomasses maximales de 580 et $780 \mathrm{mg}$ de poids $\mathrm{sec} / \mathrm{m}^{2}$ et des productions variant de 210 à $1310 \mathrm{mg}$ de poids $\mathrm{sec} / \mathrm{m}^{2}$ pour une cohorte donnée. Quant aux rapports $\mathrm{P} / \mathrm{B}$ max., ils sont semblables à ceux obtenus dans la même région pour des Chironomides lacustres (Laville 1972) et pour Sialis lutaria (Giani et Laville 1973 ). 


\section{8. - DISCUSSION}

La répartition des larves de Micrasema est influencée par le climat hydrologique général et notamment par la température (Décamps 1968). Ce phénomène, classique, correspond à la succession suivante d'amont vers l'aval : $M$. morosum - difficile minimum - longulum - moestum.

Compte tenu de cette condition première, comment expliquer la plus ou moins grande abondance des différentes espèces de Micrasema dans les mousses d'eau courante? En première analyse, l'abondance des Iarves de Micrasema est liée à l'abondance des mousses. Les larves trouvent dans ce milieu un abri et une source de nourriture, tant par les mousses elles-mêmes que par les algues qui se développent à leur surface. Aucune espèce de Micrasema ne semble inféodée à une mousse particulière. Mais certaines mousses, les Fontinalis par exemple, abritent des populations larvaires plus abondantes car elles offrent une plus grande surface d'accrochage aux larves, et partant, un plus grand nombre d'abris.

Les besoins en nourriture étant largement couverts dans les stations étudiées, la principale cause de compétition possible, interou intraspécifique, doit être at!ribuée à cette recherche d'abris. Lorsque les mousses recouvrent une surface importante du fond, comme au ruisseau d'Artigusse, à $1590 \mathrm{~m}$, ou même à la rivière le Nistos à $550 \mathrm{~m}$, des populations d'espèces différentes peuvent prospérer. De plus, dans ces stations où coexistent plusieurs espèces, les décalages observés dans les périodes de croissance réduisent fortement la compétition.

Toutefois, en une station comme le ruisseau d'Espiaube à $1360 \mathrm{~m}$, au climat hydrologique convenant à $M$. morosum, difficile et minimum, la rareté des mousses défavorise probablement les deux dernières espèces au profit de la première, mieux adapléc aux conditions locales. Une concurrence plus sévère peut donc apparâ̂tre lorsque les mousses ne recouvrent qu'une faible surface du fond. Dans le cas de l'Espiaube, cette rareté des mousses est vraisemblablement due aux très fortes turbidités provenant du ruissellement pluvial sur des rives non stabilisées. Tout facteur écologique réduisant le développement des mousses (Hynes 1970) peut avoir un effet semblable.

Fn dépit d'une relative stabilité de la couverture de mousses, comme à Artigusse, le nombre de larves peut varier considérablement d'une cohorte à l'autre pour une même espèce de Micrascma. La compétition au niveau larvaire ne paraît pas en cause; mais plutôt des conditions météorologiques différentes d'une année à l'autre, ou de prédation à un moment précis du cycle vital : méta- 
morphose, dépôt des œufs, éclosions. Ces fluctuations des nombres entraînent des variations importantes dans les biomasses et les productions d'une génération à la suivante. Mais deux constances méritent d'être soulignées :

(1) La croissance est très semblable entre les générations successives d'une même population de Micrasema. Les taux de croissance varient au sein d'une même cohorte, mais ces variations se retrouvent régulièrement d'une cohorte à la suivante. Quoique plus variable, le taux de diminution des nombres ( $\sim$ taux de mortalité) évolue aussi d'une manière semblable pour les cohortes successives de $M$. morosum ou de $M$. difficile au ruisseau d'Artigusse. L'origine des variations observées dans les nombres d'une cohorte à la suivante est donc à rechercher principalement au niveau de la séquence nymphes - adultes - pontes. Selon les années, diverses influences peuvent s'exercer sur cette période du cycle (climat). En revanche, les possibilités de régulation paraissent faibles : les larves étant relativement protégées dans leur fourreau, les invertébrés prédateurs ne s'attaquent guère qu'aux pontes. D'importantes variations sont donc susceptibles de se produire d'une cohorte à la suivante.

(2) Les rapports $\mathrm{P} / \mathrm{B}$ max. sont relativement constants pour les trois cohortes étudiées au ruisseau d'Artigusse à $1590 \mathrm{~m}$. Ils sont également semblables à ceux obtenus pour diverses larves d'insectes aquatiques du lac de Port-Bielh à $2280 \mathrm{~m}$ (Laville 1972, Giani et Laville 1973). Ce résultat peut surprendre si l'on considère la diversité des milieux et des groupes étudiés : lac, ruisseaux, Diptères Chironomides, Mégaloptères, Trichoptères. Il confirme la vraisemblance d'un $\mathrm{P} / \mathrm{B}$ max. voisin d'une valeur comprise entre 1 et 2 pour les insectes aquatiques de montagne des Pyrénées centrales. Ce rapport $\mathrm{P} / \mathrm{B}$ max. peut même être considéré comme voisin de 1,5 car la biomasse maximale de Micrasema morosum est certainement surévaluée en Artigusse. Il en résulte une sous-évaluation du rapport $\mathrm{P} / \mathrm{B}$ max. qui, pour cette espèce, s'approche probablement de 1,2 .

\section{TRAVAUX GITÉS}

Ald.en (K. R.). 1951. - The Horokiwi stream. A study of a trout population. Fish. Bull. N. Z., $10: 1-231$.

Andersson (E.). 1969. - Life cycle and growth of Asellus aquaticus (L.) with special reference to the effects of temperature. Inst. Freshwat. Res. Drottningholm Rep., $n^{\circ} 49: 5-26$.

BERTHÉlEMy (C.). 1966. - Recherches écologiques et biogéographiques sur les Plécoptères et Coléoptères d'eau courante (Hydraena et Elminthidae) des Pyrénées. Annls Limnol., 2 : 227-458. 
Dawson (F. H.). 1973. - Notes on the production of stream bryophytes in the high Pyrenees (France). Annls Limnol., $9: 231-240$.

Décamps (H.). 1967. - Écologie des Trichoptères de la Vallée d'Aure (Hautes-Pyrénées). Annls Limnol., 3 : 399-577.

DÉCAMPS (H.). 1968. - Vicariances écologiques chez les Trichoptères des Pyrénées. Annls Limnol, $4: 1-50$.

Dicanss (H.). 1970. - Les larves de Brachycentridae (Trichoptera) de la Faune de France. Taxonomie et Écologie. Annls Limnol., 6 : 51-73.

Edmondson (W. T.) et Winberg (G. G.). 1971. - A manual on methods for the assessment of secondary productivity in fresh waters. I.B.P. Handbook, n" 17, Oxford, Blackwell, 358 p.

Elliot (J.M.). 1973. - The life cycle and production of the leech Erpobdella octoculata (L.) (Hirudinea : Erpobdellidae) in a lake district stream. J. Anim. Ecol., 42 : 435-448.

Euliott (J. M.) et Décamps (H.). 1973. -- Guide pour l'analyse statistique des échantillons d'invertébrés benthiques. Annls Limnol., 9: 79120.

Frost (W. E.). 1942. - River Liffey survey IV -. The fauna of the submerged "mosses" in an acid and an alkaline water. Proc. $R$. Ir. Acad., 47 B : 293-369.

Giani (N.) et Lavirde (H.). 1973. - Cycle biologique et production de Sialis lutaria L. (Mégaloptère) dans le lac de Port-Bielh (Pyrénées Centrales). Annls Limnol., 9 : 45-61.

Hynes (H.B. N.). 1961. - The invertebrate fauna of a Welsh Mountain stream. Arch. Hydrobiol., $57: 344-388$.

Hynes (H. B. N.). 1970. - The ecology of running waters. Liverpool University Press, 555 p.

KaJaK (Z.) et Ryback (J. I.). 1966. - Production and some trophic dependances in benthos against primary production and zooplancton production of several Masurian lakes. Verh. int. Ver. Limnol., $16:$ 441-451.

Kamler (E.) et Riener. (W.). 1960. - A method for quantitative study of the bottom fauna of Tatra streams. Polskie Archwn Hydrobiol., $8: 95-105$.

LAville (H.) 1972 (1971). - Recherches sur les Chironomides (Diptera) lacustres du Massif de Néouvielle (Hautes-Pyrénées). II. Communautés et Production. Annls Limnol., 7 : 335-414.

Minckiey (W. L.). 1963. - The ecology of a spring stream Doe Run, Meade County, Kentucky. Wildl. Monogr. Cheslertown, 11 : 1-124.

Pearson (W. D.) et Kramer (R. H.). 1972. - Drift and production of two aquatic insects in a mountain stream. Ecol. Monogr., 42 (3) : $365-385$.

Ricker (W. E.). 1946. - Production and utilization of fish populations. Ecol. Monogr., 16 : 373-391.

Verneaux (J.). 1972. - La larve de Micrasema setiferum Pictet (Trichoptera, Brachycentridae). Description et données écologiques. Annls Limnol., $8:$ 49-62. 